\title{
Heavy quark expansion and universal form factors in quark model
}

\author{
Dmitri Melikhov \\ Nuclear Physics Institute, Moscow State University, Moscow, 119899, Russia
}

\begin{abstract}
Meson transition amplitudes of the vector, axial-vector, and tensor quark currents are analyzed within dispersion formulation of the constituent quark model. The form factors in the decay region are given by relativistic double spectral representations through the wave functions of the inital and final mesons. We perform heavy quark expansion of the quarkmodel mesonic matrix elements with a next-to-leading order accuracy and demonstrate that matching this expansion to the heavy-quark expansion in QCD requires subtractions in the double spectral representations for the form factors and allows fixing the subtraction terms. The Isgur-Wise function and next-to-leading order universal form factors are calculated.
\end{abstract}

\section{INTRODUCTION}

Theoretical description of hadronic amplitudes of the quark currents is one of the key problems of particle physics as such amplitudes provide a bridge between QCD formulated in the language of quarks and gluons and observable phenomena which deal with hadrons. In particular, the knowledge of such amplitudes is necessary for extraction of the parameters of the quark-mixing matrix in the Standard Model from the experiments on weak heavy hadron decays. The difficulty in such calculations lie in the fact that hadron formation occurs at large distances where perturbative QCD methods are not applicable and nonperturbative consideration is necessary.

Various theoretical frameworks more or less directly related to QCD have been applied to the description of meson transition form factors: among them constituent quark models [1 15], lattice QCD simulations [16 18], QCD sum rules 19 25], analytical constraints [26,27].

Lattice QCD simulations is the most direct QCD based nonperturbative approach and thus should in principle provide most reliable results. However it faces serious technical problems with placing heavy particles on the lattice and performing calculations with inclusion of quark loops. So far direct calculations with $b$-quark are not possible and extrapolation in the quark mass is used which considerably reduces the predictive power of the method.

Various versions of QCD sum rules to meson decays give very uncertain predictions strongly dependent on the technical subtleties of the particular version. A recent analysis [23] disregards the three-point QCD sum rules in favor of the light-cone sun rules which however involve more phenomenological inputs.

Constituent quark models have proved to be a fruitful phenomenological method for the description of heavy meson transition form factors. Constituent quark models employ the fact that heavy meson consists of a heavy quark and light degrees of freedom with have quantum numbers of the quark state and assume that these light states can be approximately described by an effective constituent quark. The application of the various versions of the constituent quark picture to the decay processes has a long history. The first models were based on a semirelativistic [1] or nonrelativistic [2, 4 considerations and didn't treat the quark spins thoroughly. Further developments demonstrated the relativistic description of the quark spins to be important for consistent applications to meson decays. The exact solution to a complicated dynamical problem of treating the spins of the interacting particles is not known, but a simplified self-consistent relativistic consideration of the quark spins can be performed within the light-cone quark model (LCQM) [6]. The difficulty with the application of this approach to the decay processes lies in the fact that the applicability of the model is restricted by the condition $q^{2} \leq 0$, while the physical region for hadron decays is $0 \leq q^{2} \leq\left(M_{i}-M_{f}\right)^{2}, M_{i, f}$ being the initial and final hadron masses, respectively. The problem is connected with the contribution of the so-called pair-creation subprocesses which cannot be taken into account thoroughly in the model, except for a trivial case of a pointlike interaction. At spacelike momentum transfers the contribution of these subprocesses can be killed by choosing an appropriate reference frame, whereas at timelike momentum transfers such a frame does not exist and thus pair creation contributes together with the partonic contribution. An estimate of the form factors at timelike momentum transfers within the LCQM has been done in [6] where the transition form factors have been calculated in the spacelike region and then numerically extrapolated to the timelike region assuming some particular $q^{2}$-behavior. This works well if the accessible momentum transfer interval is not large. However, in the heavy-to-light meson decays this interval is of order of the heavy meson mass squared and the extrapolation procedure yields large uncertainties. The approach of refs [11 13 calculates the partonic contribution to the form factors at timelike momentum transfers and thus avoids extrapolations. However, the nonpartonic contribution is omitted. Such treatment can be justified if the latter is small that is not obvious a priori. Thus, one can see that for a reliable description of the decay processes within the LCQM it might be 
reasonable to find another formulation of the model appropriate also at timelike momentum transfers. Such a formulation has been proposed in 14 .

The approach of 114 is based on the dispersion formulation of the LCQM. Namely, the transition form factors obtained within the light-cone formalism at $q^{2}<0$ [6], are represented as dispersion integrals over initial and final hadron masses through their light-cone wave functions. The spectral densities of these representations can be calculated from the Feynman graphs. The transition form factors at $q^{2}>0$ are derived by performing the analytical continuation in $q^{2}$ from the region $q^{2} \leq 0$. As a result, for a decay caused by the weak transition of the quark $Q\left(m_{i}\right) \rightarrow Q\left(m_{f}\right)$, form factors in the region $q^{2} \leq\left(m_{i}-m_{f}\right)^{2}$ are expressed through the light-cone wave functions of the initial and final hadrons and can be calculated in the decay region avoiding dangerous extrapolations.

At the same time, such a dispersion formulation of the LCQM allows also a bit different view on the problem of constructing the form factors within the quark model: actually, we calculate the double spectral densities from the Feynman graphs and thus obtain unsubtracted spectral representations. On the other hand we know that in general subtractions might be added to such representations and we need some additional arguments to decide on the necessity and the structure of the subtraction terms.

Let us notice that once we are working within an approach not directly deduced from QCD it is important to preserve essential features of the underlying fundamental theory in the model. Thus matching the results obtained within the quark model to rigorous QCD results might be helpful for bringing more realistic features to the model. We use such matching to QCD results for determining the subtraction terms in the double spectral representations of the form factors.

There are few QCD predictions on meson form factors in the decay region. Fortunately, there is an important case in which QCD provides rigorous and model-independent results on transition form factors: namely, meson decays induced by transitions between heavy quarks. In this case the heavy-quark symmetry simplifies all the form factors in the leading $1 / m_{Q}$ order to the universal Isgur-Wise function [28]. A systematic expansion in inverse powers of the heavy quark mass can be constructed within the Heavy quark effective theory [31] based on QCD with the heavy quarks. Although the form factors appearing in different $1 / m_{Q}$ orders can not be determined within the HQET, the latter restricts the structure of the expansion and the number of these universal form factors in each $1 / m_{Q}$ order and provides constraints on some of them at zero recoil.

The idea of matching the quark model form factors to the structure of the heavy-quark expansion of HQET has been employed in the ISGW2 model [5]. However the ISGW2 model does not calculate the form factors within a relativistic dynamical approach on the basis of considering the meson structure but rather gives prescription for constructing the form factors. The approach of ref. [7] also employs matching to the heavy-quark expansion and obtains interesting results within the constutuent quark picture assuming a strong peaking of the momentum distribution of the constituent quarks inside a meson and avoiding details of the dynamics of the process. This model imposes constraints on the form factors but does not allow one to calculate them.

The idea implemented in this work is to perform the heavy-quark expansion in the dispersion formulation of the quark model and to match this expansion to the HQET. This matching is used for determining the structure of the subtraction terms in the spectral representations. We consider the form factors of pseudoscalar meson transitions into pseudoscalar and vector final mesons through the vector, axial-vector, and currents and analyze the expansion in the leading and next-to-leading order. Further constraints on the subtraction terms are obtained by considering the case of the heavy-to-light quark transition and analyzing the relations between the form factors of the tensor, vector and axial-vector currents. Comparing the relations obtained within the dispersion quark model to the general relations found by Isgur and Wise 32 allows us to fix the subtraction terms.

Our main results are the following:

1. Applying the approach of [29] to heavy meson transition throught the tensor current, we derive the decomposition of the form factors $h_{g_{+}}, h_{g_{-}}, h_{g_{0}}$, and $h_{s}$ (the definitions are given in the next section) in the next-to-leading order in $1 / m_{Q}$. In particular, we have found that the $1 / m_{Q}$ corrections to the form factor $h_{g_{+}}$ at zero recoil vanish just as for the form factors $h_{f_{+}}$and $h_{f}$ (the Luke theorem). Although the heavy meson transitions through the tensor current do not correspond to a real experimental situation we need the expansion for such form factors as a bench mark for comparison of the quark model.

2. We present an improved dispersion formulation of the LCQM: the transition form factors of pseudoscalar $(P)$ mesons into pseudoscalar $(P)$ and vector $(V)$ mesons induced by the vector, axial-vector and tensor currents are given by double spectral representations through the wave functions of the initial and final mesons. The unsubtracted double spectral densities are calculated from the Feynman graphs and the subtraction terms in the spectral representations are determined by matching the $1 / m_{Q}$ expansion in the quark model to the HQET. 3. We perform the heavy-quark expansion of the form factors of the dispersion quark model up to NLO and calculate the Isgur-Wise function and the NLO universal form factors assuming a strong peaking of the soft wave function near the $q \bar{q}$ threshold with a width of order $\Lambda_{Q C D}$. We obtain in the LCQM the relations

$$
\chi_{2}(\omega)=0 ; \quad \chi_{3}(\omega)=0 ; \quad \xi_{3}(\omega)>0, \xi_{3}(1)=\langle z\rangle / 3,
$$


where $\langle z\rangle$ is an average kinetic energy of the light quark in the heavy meson rest frame.

We observe that matching the $1 / m_{Q}$ expansion of the quark model to HQET in LO and NLO requires subtractions in some of the form factors describing the $P \rightarrow V$ transition and restricts the form of the subtraction terms.

4. We consider the heavy-to-light meson transitions in which case a small parameter $\Lambda_{Q C D} / m_{Q}$ emerges and analyze the form factors in the leading $\Lambda_{Q C D} / m_{Q}$ order. Requiring the fulfillment of the Isgur-Wise relatins for the heavy-to-light transitions [32] further constrains the subtraction terms providing explicit spectral representations with subtractions for the form factors of interest.

In the next section we remind the definitions of the form factors and list the HQET results for the amplitudes of the $P \rightarrow P$ and $P \rightarrow V$ transitions through the vector, axial-vector and tensor currents up to NLO. In section 3 we present the quark-model results for the meson decay form factors at arbitrary masses: namely, we calculate the unsubtracted spectral densities and explicit form of the subtraction terms which form is motivated in the subsequent section. In Section 4 the $1 / m_{Q}$ expansions of the wave functions and the form factors in quark model for the heavy-to-light transitions are considered. In Section 5 the heavy-to-light case is discussed. Section 6 illustrates the main results with numerical estimates and evaluate the Isgur-Wise function for various quark model parameters. Conclusion summarizes the results.

\section{MESON TRANSITION AMPLITUDES AND HEAVY-QUARK EXPANSION IN QCD}

The amplitudes of meson decays induced by the quark transition $q_{2} \rightarrow q_{1}$ through the vector $V_{\mu}=\bar{q}_{1} \gamma_{\mu} q_{2}$, axial-vector $A_{\mu}=\bar{q}_{1} \gamma_{\mu} \gamma_{5} q_{2}$, and tensor $T_{\mu \nu}=\bar{q}_{1} \sigma_{\mu \nu} q_{2}$ currents have the following structure 32

$$
\begin{aligned}
<P\left(M_{2}, p_{2}\right)\left|V_{\mu}(0)\right| P\left(M_{1}, p_{1}\right)> & =f_{1}\left(q^{2}\right) p_{1 \mu}+f_{2}\left(q^{2}\right) p_{2 \mu}, \\
<V\left(M_{2}, p_{2}, \epsilon\right)\left|V_{\mu}(0)\right| P\left(M_{1}, p_{1}\right)> & =2 g\left(q^{2}\right) \epsilon_{\mu \nu \alpha \beta} \epsilon^{* \nu} p_{1}^{\alpha} p_{2}^{\beta}, \\
<V\left(M_{2}, p_{2}, \epsilon\right)\left|A_{\mu}(0)\right| P\left(M_{1}, p_{1}\right)> & =i \epsilon^{* \alpha}\left[f\left(q^{2}\right) g_{\mu \alpha}+a_{1}\left(q^{2}\right) p_{1 \alpha} p_{1 \mu}+a_{2}\left(q^{2}\right) p_{1 \alpha} p_{2 \mu}\right], \\
<P\left(M_{2}, p_{2}\right)\left|T_{\mu \nu}(0)\right| P\left(M_{1}, p_{1}\right)> & =-2 i s\left(q^{2}\right)\left(p_{1 \mu} p_{2 \nu}-p_{1 \nu} p_{2 \mu}\right), \\
<V\left(M_{2}, p_{2}, \epsilon\right)\left|T_{\mu \nu}(0)\right| P\left(M_{1}, p_{1}\right)> & =i \epsilon^{* \alpha}\left[g_{1}\left(q^{2}\right) \epsilon_{\mu \nu \alpha \beta} p^{1 \beta}+g_{2}\left(q^{2}\right) \epsilon_{\mu \nu \alpha \beta} p^{2 \beta}+g_{0}\left(q^{2}\right) p_{1 \alpha} \epsilon_{\mu \nu \beta \gamma} p_{1}^{\beta} p_{2}^{\gamma}\right],
\end{aligned}
$$

with $q=p_{1}-p_{2}, P=p_{1}+p_{2}$. We use the notations: $\gamma^{5}=i \gamma^{0} \gamma^{1} \gamma^{2} \gamma^{3}, \sigma_{\mu \nu}=\frac{i}{2}\left[\gamma_{\mu}, \gamma_{\nu}\right], \epsilon^{0123}=-1$, and $S p\left(\gamma^{5} \gamma^{\mu} \gamma^{\nu} \gamma^{\alpha} \gamma^{\beta}\right)=4 i \epsilon^{\mu \nu \alpha \beta}$.

The relativistic-invariant form factors contain the dynamical information on the process and should be calculated within a nonperturbative approach for any particular initial and final mesons.

For analysing the transition in the case when both the parent and the daughter quarks inducing the meson transition are heavy, i.e. $m_{1} \simeq m_{2} \gg \Lambda_{Q C D}$ it is convenient to introduce a new dimensionless variable $\omega=v_{1} v_{2}=\frac{M_{1}^{2}+M_{2}^{2}-q^{2}}{M_{1} M_{2}}$ and velocity-dependent form factors connected with 4-velocities and not 4-momenta as in (2) in the following way

$$
\begin{aligned}
<P\left(M_{2}, p_{2}\right)\left|V_{\mu}(0)\right| P\left(M_{1}, p_{1}\right)>= & \sqrt{M_{1} M_{2}}\left[h_{f_{+}}(\omega)\left(v_{1}+v_{2}\right)_{\mu}+h_{f_{-}}(\omega)\left(v_{1}-v_{2}\right)_{\mu}\right], \\
<V\left(M_{2}, p_{2}, \epsilon\right)\left|V_{\mu}(0)\right| P\left(M_{1}, p_{1}\right)>= & \sqrt{M_{1} M_{2}} h_{g}(\omega) \epsilon_{\mu \nu \alpha \beta} \epsilon^{* \nu} v_{1}^{\alpha} v_{2}^{\beta}, \\
<V\left(M_{2}, p_{2}, \epsilon\right)\left|A_{\mu}(0)\right| P\left(M_{1}, p_{1}\right)>= & i \epsilon^{* \alpha} \sqrt{M_{1} M_{2}}\left[h_{f}(\omega)(1+\omega) g_{\mu \alpha}-h_{a_{1}}(\omega) v_{1 \alpha} v_{1 \mu}-h_{a_{2}}(\omega) v_{1 \alpha} v_{2 \mu}\right], \\
<P\left(M_{2}, p_{2}\right)\left|T_{\mu \nu}(0)\right| P\left(M_{1}, p_{1}\right)>= & -2 i \sqrt{M_{1} M_{2}} h_{s}(\omega)\left(v_{1 \mu} v_{2 \nu}-v_{1 \nu} v_{2 \mu}\right), \\
<V\left(M_{2}, p_{2}, \epsilon\right)\left|T_{\mu \nu}(0)\right| P\left(M_{1}, p_{1}\right)>= & i \epsilon^{* \alpha} \sqrt{M_{1} M_{2}}\left[h_{g_{+}}(\omega) \epsilon_{\mu \nu \alpha \beta}\left(v_{1}+v_{2}\right)^{\beta}+h_{g_{-}}(\omega) \epsilon_{\mu \nu \alpha \beta}\left(v_{1}-v_{2}\right)^{\beta}\right. \\
& \left.+h_{g_{0}}(\omega) v_{1 \alpha} \epsilon_{\mu \nu \beta \gamma} v_{1}^{\beta} v_{2}^{\gamma}\right] .
\end{aligned}
$$

These form factors are related to the form factors introduced by the relations (2) as follows

$$
\begin{aligned}
f_{1} & =\frac{M_{2}}{\sqrt{M_{1} M_{2}}}\left[h_{f_{+}}+h_{f_{-}}\right], \quad g_{1}=\frac{M_{2}}{\sqrt{M_{1} M_{2}}}\left[h_{g_{+}}+h_{g_{-}}\right], \\
f_{2} & =\frac{M_{1}}{\sqrt{M_{1} M_{2}}}\left[h_{f_{+}}-h_{f_{-}}\right], \quad g_{2}=\frac{M_{1}}{\sqrt{M_{1} M_{2}}}\left[h_{g_{+}}-h_{g_{-}}\right], \\
g & =\frac{1}{2 \sqrt{M_{1} M_{2}}} h_{g}, \quad s=\frac{1}{2 \sqrt{M_{1} M_{2}}} h_{s}, \\
f & =\sqrt{M_{1} M_{2}}(1+\omega) h_{f}, \quad a_{2}=-\frac{1}{\sqrt{M_{1} M_{2}}} h_{a_{2}}, \quad a_{1}=-\frac{1}{\sqrt{M_{1} M_{2}}} \frac{M_{2}}{M_{1}} h_{a_{1}}, \quad g_{0}=\frac{1}{\sqrt{M_{1} M_{2}}} \frac{1}{M_{1}} h_{g_{0}}
\end{aligned}
$$


The form factors $h$ are convenient quantities as in the leading $1 / m_{Q}$ order all of them are expressed through a single universal function of the dimensionless variable $\omega$ - the Isgur-Wise function. A consistent heavy-quark expansion of the form factors, i.e. expansion in inverse powers of the heavy-quark mass, can be constructed within the Heavy Quark Effective Theory based on QCD with heavy quarks.

The general structure of the $1 / m_{Q}$ expansion of the heavy quark form factors in QCD for the meson transition $M_{1} \rightarrow M_{2}$ induced by heavy quark transition $m_{2} \rightarrow m_{1}$ have the form (omitting corrections $O\left(\alpha_{s}, \alpha_{s} / m_{Q}, 1 / m_{Q}^{2}\right)$ :

$$
\begin{aligned}
& h_{f_{+}}=\xi+\left(\frac{1}{m_{1}}+\frac{1}{m_{2}}\right) \rho_{1}, \\
& h_{f_{-}}=\quad\left(\frac{1}{m_{1}}-\frac{1}{m_{2}}\right)\left(-\frac{\bar{\Lambda}}{2} \xi+\xi_{3}\right) \text {, } \\
& h_{g}=\xi+\left(\frac{1}{m_{1}}+\frac{1}{m_{2}}\right) \frac{\bar{\Lambda}}{2} \xi+\frac{1}{m_{1}} \rho_{2}+\frac{1}{m_{2}}\left(\rho_{1}-\xi_{3}\right), \\
& h_{f}=\xi+\left(\frac{1}{m_{1}}+\frac{1}{m_{2}}\right) \frac{\omega-1}{\omega+1} \frac{\bar{\Lambda}}{2} \xi+\frac{1}{m_{1}} \rho_{2}+\frac{1}{m_{2}}\left(\rho_{1}-\frac{\omega-1}{\omega+1} \xi_{3}\right), \\
& h_{a_{1}}=\quad \frac{1}{m_{1}} \frac{1}{\omega+1}\left(-\bar{\Lambda} \xi+2(\omega+1) \chi_{2}-\xi_{3}\right) \text {, } \\
& h_{a_{2}}=\xi+\left(\frac{\omega-1}{\omega+1} \frac{1}{m_{1}}+\frac{1}{m_{2}}\right) \frac{\bar{\Lambda}}{2} \xi+\frac{1}{m_{1}}\left(\rho_{2}-2 \chi_{2}-\frac{1}{\omega+1} \xi_{3}\right)+\frac{1}{m_{2}}\left(\rho_{1}-\xi_{3}\right) . \\
& h_{s}=\xi+\left(\frac{1}{m_{1}}+\frac{1}{m_{2}}\right)\left(\frac{\bar{\Lambda}}{2} \xi-\xi_{3}+\rho_{1}\right) \text {, } \\
& h_{g_{+}}=-\xi-\frac{1}{m_{2}} \rho_{1}-\frac{1}{m_{1}} \rho_{2}, \\
& h_{g_{-}}=\quad\left(\frac{1}{m_{1}}-\frac{1}{m_{2}}\right) \frac{\bar{\Lambda}}{2} \xi+\frac{1}{m_{2}} \xi_{3}, \\
& h_{g_{0}}=\quad \frac{1}{m_{1}}\left(\frac{\bar{\Lambda} \xi+\xi_{3}}{\omega+1}+2 \chi_{2}\right) \text {. }
\end{aligned}
$$

In the leading $1 / m_{Q}$ order (LO) all the form factors are represented through the single universal Isgur-Wise function $\xi(\omega)$, whereas in the next-to-leading order (NLO) the 4 new form factors $\rho_{1}, \rho_{2}, \chi_{2}$, and $\xi_{3}$ appear. The universal form factors are functions of a single variable $\omega$.

The form factor $\xi_{3}$ originates from the expansion of the transition quark current, and the form factors $\rho_{1}, \rho_{2}, \chi_{2}$ are connected with the nontrivial relationship between the mesonic states in the full and the effective theory. The universal form factors satisfy the conditions

$$
\xi(1)=1, \quad \rho_{1}(1)=\rho_{2}(1)=0,
$$

whereas no constraints on $\xi_{3}$ and $\chi_{2}$ are imposed by the heavy quark symmetry. As found by Luke [29], the $1 / m_{Q}$ corrections to the form factors $h_{f}$ and $h_{f_{+}}$vanish due to kinematical or dynamical reasons. One can see that the same is true for the form factor $h_{g_{+}}$: namely,

$$
h_{g_{+}}(1)=-1+O\left(1 / m_{Q}^{2}\right)
$$

The parameter $\bar{\Lambda}$ in (5) comes from the $1 / m_{Q}$ expansion of the mass of a meson consisting of the heavy quark and light degrees of freedom

$$
M_{Q}=m_{Q}+\bar{\Lambda}+O\left(1 / m_{Q}\right) .
$$

In our notations for heavy quarks and mesons, this gives

$$
M_{1}=m_{2}+\bar{\Lambda}+\ldots, \quad M_{2}=m_{1}+\bar{\Lambda}+\ldots,
$$

for the parent and daughter particles, respectively.

It is straightforward to derive the following useful relations

$$
\frac{M_{1}+M_{2}}{\sqrt{M_{1} M_{2}}}=\frac{m_{2}+m_{1}}{\sqrt{m_{1} m_{2}}}\left[1-\left(\frac{1}{m_{1}}+\frac{1}{m_{2}}\right)\left(\frac{m_{2}-m_{1}}{m_{2}+m_{1}}\right)^{2} \frac{\bar{\Lambda}}{2}+\ldots\right]
$$




$$
\begin{aligned}
\frac{M_{1}-M_{2}}{\sqrt{M_{1} M_{2}}}=\frac{m_{2}-m_{1}}{\sqrt{m_{1} m_{2}}}\left[1-\left(\frac{1}{m_{1}}+\frac{1}{m_{2}}\right) \frac{\bar{\Lambda}}{2}+\ldots\right] \\
\sqrt{M_{1} M_{2}}=\sqrt{m_{1} m_{2}}\left[1+\left(\frac{1}{m_{1}}+\frac{1}{m_{2}}\right) \frac{\bar{\Lambda}}{2}+\ldots\right],
\end{aligned}
$$

where the dots denote higher order terms.

Using the relations (4) and (10), we obtain for the form factors (2) the following expansions

$$
\begin{aligned}
& f_{1}=\frac{m_{1}}{\sqrt{m_{1} m_{2}}}\left[\xi+\frac{1}{m_{1}}\left(\rho_{1}+\xi_{3}\right)+\frac{1}{m_{2}}\left(\rho_{1}-\xi_{3}\right)\right] \text {, } \\
& f_{2}=\frac{m_{2}}{\sqrt{m_{1} m_{2}}}\left[\xi+\frac{1}{m_{1}}\left(\rho_{1}-\xi_{3}\right)+\frac{1}{m_{2}}\left(\rho_{1}+\xi_{3}\right)\right] \text {, } \\
& s=\frac{1}{2 \sqrt{m_{1} m_{2}}}\left[\xi+\frac{1}{m_{1}}\left(\rho_{1}-\xi_{3}\right)+\frac{1}{m_{2}}\left(\rho_{1}-\xi_{3}\right)\right], \\
& g_{1}=-\frac{m_{1}}{\sqrt{m_{1} m_{2}}}\left[\xi+\frac{1}{m_{1}} \rho_{2}+\frac{1}{m_{2}}\left(\rho_{1}-\xi_{3}\right)\right] \text {, } \\
& g_{2}=-\frac{m_{2}}{\sqrt{m_{1} m_{2}}}\left[\xi+\frac{1}{m_{1}} \rho_{2}+\frac{1}{m_{2}}\left(\rho_{1}+\xi_{3}\right)\right] \text {, } \\
& g=\frac{1}{2 \sqrt{m_{1} m_{2}}}\left[\xi+\frac{1}{m_{1}} \rho_{2}+\frac{1}{m_{2}}\left(\rho_{1}-\xi_{3}\right)\right] \text {, } \\
& a_{1}=-\frac{1}{\sqrt{m_{1} m_{2}}} \frac{1}{m_{2}} \frac{1}{\omega+1}\left[-\bar{\Lambda} \xi+2(\omega+1) \chi_{2}-\xi_{3}\right] \text {, } \\
& a_{2}=-\frac{1}{\sqrt{m_{1} m_{2}}}\left[\xi+\frac{1}{m_{1}}\left(\rho_{1}-\xi_{3}\right)+\frac{1}{m_{2}}\left(\rho_{1}-\xi_{3}\right)-\frac{\bar{\Lambda}}{m_{1}} \frac{1}{\omega+1} \xi-\frac{2 \chi_{2}}{m_{1}}+\frac{1}{m_{1}} \frac{\omega}{\omega+1} \xi_{3}\right] \text {, } \\
& f=\sqrt{m_{1} m_{2}}(\omega+1)\left[\xi+\frac{\bar{\Lambda} \xi \omega}{\omega+1}\left(\frac{1}{m_{1}}+\frac{1}{m_{2}}\right)+{\frac{1}{m_{1}}}_{1} \rho_{2}+\frac{1}{m_{2}}\left(\rho_{1}-\frac{\omega-1}{\omega+1} \xi_{3}\right)\right] \\
& g_{0}=\frac{1}{\left(m_{1} m_{2}\right)^{3 / 2}}\left[\frac{\bar{\Lambda} \xi+\xi_{3}}{\omega+1}+2 \chi_{2}\right]
\end{aligned}
$$

For the following analysis it is worth noting that the behavior of the combination $2 p_{1} p_{2} \cdot g-m_{1} \cdot g_{2}$ and $f$ in LO and NLO coincide, namely

$$
f \simeq 2 p_{1} p_{2} \cdot g-m_{1} \cdot g_{2}
$$

It is also convenient to introduce the form factor $a_{2}^{\prime}$ such that

$$
a_{2}^{\prime}=a_{2}+2 s
$$

In what follows we need the expansions of the following linear combinations of the form factors $a_{2}^{\prime}$ and $a_{1}$

$$
\begin{aligned}
& a_{1} m_{2}-a_{2}^{\prime} m_{1}=-\frac{1}{\sqrt{m_{1} m_{2}}}\left[4 \chi_{2}-\xi_{3}\right], \\
& a_{1} m_{2}+a_{2}^{\prime} m_{1}=-\frac{1}{\sqrt{m_{1} m_{2}}}\left[-\frac{2 \bar{\Lambda} \xi}{\omega+1}+\xi_{3} \frac{\omega-1}{\omega+1}\right] .
\end{aligned}
$$

\section{TRANSITION FORM FACTORS IN THE DISPERSION QUARK MODEL}

The results presented in the previous section are strict consequences of QCD in the heavy-quark limit which however cannot provide more information on the universal form factors $\xi$ and $\rho_{1}, \rho_{2}, \chi_{2}, \xi_{3}$. They must be calculated within a nonperturbative dynamical approach. We study the form factors within the dispersion formulation of the quark model [14,15] which has proved to be a reasonable framework for describing meson decays. We start with $q^{2}<0$ and represent the form factors as double spectral representations in the invariant masses of the initial and final $q \bar{q}$ pairs. The form factors at $q^{2}>0$ are derived by performing the analytical continuation. 
The transition of the initial meson $q\left(m_{2}\right) \bar{q}\left(m_{3}\right)$ with the mass $M_{1}$ to the final meson $q\left(m_{1}\right) \bar{q}\left(m_{3}\right)$ with the mass $M_{2}$ induced by the quark transition $m_{2} \rightarrow m_{1}$ through the current $\bar{q}\left(m_{1}\right) J_{\mu} q\left(m_{2}\right)$ is described by the diagram of Fig.11. For constructing the double spectral representation we must consider a double-cut graph where all intermediate particles go on mass shell but the initial and final mesons have the off-shell momenta $\tilde{p}_{1}$ and and $\tilde{p}_{2}$ such that $\tilde{p}_{1}^{2}=s_{1}$ and $\tilde{p}_{2}^{2}=s_{2}$ with $\left(\tilde{p}_{1}-\tilde{p}_{2}\right)^{2} \equiv s_{3}=q^{2}$ kept fixed.

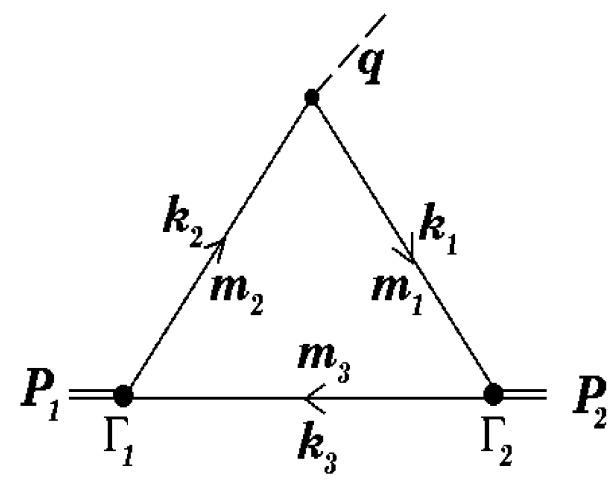

FIG. 1. One-loop graph for a meson decay.

For the transition $B \rightarrow D, D^{*} m_{2}=m_{b}, m_{1}=m_{c}$, and $m_{3}=m_{u}$. The constituent quark structure of the initial and final mesons is given in terms of the vertices $\Gamma_{1}$ and $\Gamma_{2}$, respectively. The initial pseudoscalar meson vertex has the spinorial structure $\Gamma_{1}=i \gamma_{5} G_{1} / \sqrt{N_{c}}$; the final meson vertex has the structure $\Gamma_{2}=i \gamma_{5} G_{2} / \sqrt{N_{c}}$ for a pseudoscalar state and the structure $\Gamma_{2 \mu}=\left[A \gamma_{\mu}+B\left(k_{1}-k_{3}\right)_{\mu}\right] G_{2} / \sqrt{N_{c}}, A=-1, B=1 /\left(\sqrt{s_{2}}+m_{1}+m_{3}\right)$ for an $S$-wave vector meson.

The double spectral densities $\tilde{f}$ of the form factors are obtained by calculating the relevant traces and isolating the Lorentz structures depending on $\tilde{p}_{1}$ and $\tilde{p}_{2}$. The invariant factors of such Lorentz structures provide the double spectral densities $\tilde{f}$ corresponding to taking into account contributions of the two-particle singularities in the Feynman graph. Let us point out that this procedure allows one to obtain unsubtracted double spectral densities, whereas subtraction terms should be determined independently. In this paper we determine the subtraction terms from matching the $1 / m_{Q}$ expanded form factors of the quark model to the heavy-quark expansion in QCD.

At $q^{2}<0$ the spectral representations of the form factors have the form 14]

$$
f_{i}\left(q^{2}\right)=\frac{1}{16 \pi^{2}} \int_{\left(m_{1}+m_{3}\right)^{2}}^{\infty} d s_{2} \varphi_{2}\left(s_{2}\right) \int_{s_{1}^{-}\left(s_{2}, q^{2}\right)}^{s_{1}^{+}\left(s_{2}, q^{2}\right)} d s_{1} \varphi_{1}\left(s_{1}\right) \frac{\tilde{f}_{i}\left(s_{1}, s_{2}, q^{2}\right)}{\lambda^{1 / 2}\left(s_{1}, s_{2}, q^{2}\right)},
$$

where the wave function $\varphi_{i}\left(s_{i}\right)=G_{i}\left(s_{i}\right) /\left(s_{i}-M_{i}^{2}\right)$ and

$$
s_{1}^{ \pm}\left(s_{2}, q^{2}\right)=\frac{s_{2}\left(m_{1}^{2}+m_{2}^{2}-q^{2}\right)+q^{2}\left(m_{1}^{2}+m_{3}^{2}\right)-\left(m_{1}^{2}-m_{2}^{2}\right)\left(m_{1}^{2}-m_{3}^{2}\right)}{2 m_{1}^{2}} \pm \frac{\lambda^{1 / 2}\left(s_{2}, m_{3}^{2}, m_{1}^{2}\right) \lambda^{1 / 2}\left(q^{2}, m_{1}^{2}, m_{2}^{2}\right)}{2 m_{1}^{2}}
$$

and $\lambda\left(s_{1}, s_{2}, s_{3}\right)=\left(s_{1}+s_{2}-s_{3}\right)^{2}-4 s_{1} s_{2}$ is the triangle function.

The unsubtracted double spectral densities $\tilde{f}_{i}\left(s_{1}, s_{2}, q^{2}\right)$ of the form factors read [14, 15]:

$$
\begin{aligned}
\tilde{s} & =2\left[m_{1} \alpha_{2}+m_{2} \alpha_{1}+m_{3}\left(1-\alpha_{1}-\alpha_{2}\right)\right], \\
\tilde{f}_{1} & =2 m_{1} \tilde{s}+4 \alpha_{2}\left[s_{2}-\left(m_{1}-m_{3}\right)^{2}\right]-2 m_{3} \tilde{s}, \\
\tilde{f}_{2} & =2 m_{2} \tilde{s}+4 \alpha_{1}\left[s_{1}-\left(m_{2}-m_{3}\right)^{2}\right]-2 m_{3} \tilde{s}, \\
\tilde{g} & =A \tilde{s}-4 B \beta \\
\tilde{g}_{1} & =A \tilde{f}_{1}-8 \beta+8 B\left(m_{1}+m_{3}\right) \beta, \\
\tilde{g}_{2} & =A \tilde{f}_{2}+8 B\left(m_{2}-m_{3}\right) \beta \\
\tilde{a}_{2 D} & =-2 \tilde{s}+4 B C_{2} \alpha_{1}+\alpha_{12} C_{0}, \\
\tilde{a}_{1 D} & =-4 A\left(2 m_{3}+B C_{1}\right) \alpha_{1}+\alpha_{11} C_{0}, \\
\tilde{f}_{D} & =-4 A\left[m_{1} m_{2} m_{3}+\frac{m_{2}}{2}\left(s_{2}-m_{1}^{2}-m_{3}^{2}\right)+\frac{m_{1}}{2}\left(s_{1}-m_{2}^{2}-m_{3}^{2}\right)-\frac{m_{3}}{2}\left(s_{3}-m_{1}^{2}-m_{2}^{2}\right)\right]+C_{0} \beta, \\
\tilde{g}_{0 D} & =-8 A \alpha_{12}-8 B\left[-m_{3} \alpha_{1}+\left(m_{3}-m_{2}\right) \alpha_{11}+\left(m_{3}+m_{1}\right) \alpha_{12}\right],
\end{aligned}
$$


where

$$
\begin{aligned}
\alpha_{1} & =\left[\left(s_{1}+s_{2}-s_{3}\right)\left(s_{2}-m_{1}^{2}+m_{3}^{2}\right)-2 s_{2}\left(s_{1}-m_{2}^{2}+m_{3}^{2}\right)\right] / \lambda\left(s_{1}, s_{2}, s_{3}\right), \\
\alpha_{2} & =\left[\left(s_{1}+s_{2}-s_{3}\right)\left(s_{1}-m_{2}^{2}+m_{3}^{2}\right)-2 s_{1}\left(s_{2}-m_{1}^{2}+m_{3}^{2}\right)\right] / \lambda\left(s_{1}, s_{2}, s_{3}\right), \\
\beta & =\frac{1}{4}\left[2 m_{3}^{2}-\alpha_{1}\left(s_{1}-m_{2}^{2}+m_{3}^{2}\right)-\alpha_{2}\left(s_{2}-m_{1}^{2}+m_{3}^{2}\right)\right], \\
\alpha_{11} & =\alpha_{1}^{2}+4 \beta s_{2} / \lambda\left(s_{1}, s_{2}, s_{3}\right), \quad \alpha_{12}=\alpha_{1} \alpha_{2}-2 \beta\left(s_{1}+s_{2}-s_{3}\right) / \lambda\left(s_{1}, s_{2}, s_{3}\right), \\
C_{0} & =-8 A\left(m_{2}-m_{3}\right)+4 B C_{3}, \quad C_{1}=s_{2}-\left(m_{1}+m_{3}\right)^{2}, \\
C_{2} & =s_{1}-\left(m_{2}-m_{3}\right)^{2}, \quad C_{3}=s_{3}-\left(m_{1}+m_{2}\right)^{2}-C_{1}-C_{2} .
\end{aligned}
$$

We label with a subscript ' $\mathrm{D}$ ' the double spectral densities of the form factors which require subtractions. We fix this subtraction procedure by requiring the $1 / m_{Q}$ expansion of the form factors to have a proper form in accordance with QCD in leading and next-to-leading orders for the case of a meson transition caused by heavy-to-heavy quark transition. As we shall see this yields the double spectral densities which include properly defined subtraction terms

$$
\begin{aligned}
& \tilde{f}=\tilde{f}_{D}+\left[\left(M_{1}^{2}-s_{1}\right)+\left(M_{2}^{2}-s_{2}\right)\right] \tilde{g}, \\
& \tilde{a}_{1}=\tilde{a}_{1 D}+\frac{1}{(\bar{\omega}+1) m_{2}}\left(\frac{M_{1}^{2}-s_{1}}{\sqrt{s_{1}}}+\frac{M_{2}^{2}-s_{2}}{\sqrt{s_{2}}}\right) \frac{\tilde{g}}{2}, \\
& \tilde{a}_{2}=\tilde{a}_{2 D}+\frac{1}{(\bar{\omega}+1) m_{1}}\left(\frac{M_{1}^{2}-s_{1}}{\sqrt{s_{1}}}+\frac{M_{2}^{2}-s_{2}}{\sqrt{s_{2}}}\right) \frac{\tilde{g}}{2}, \\
& \tilde{g}_{0}=\tilde{g}_{0 D}+\frac{1}{(\bar{\omega}+1) m_{1} m_{2}}\left(\frac{M_{1}^{2}-s_{1}}{\sqrt{s_{1}}}+\frac{M_{2}^{2}-s_{2}}{\sqrt{s_{2}}}\right) \frac{\tilde{g}}{2} .
\end{aligned}
$$

As the analytical continuation to the timelike region is performed, in addition to the normal contribution which is just the expression (25) taken at $q^{2}>0$, anomalous contribution emerges [19]. The origin of the anomalous contribution is connected with the motion of a zero of the triangle function $\lambda\left(s_{1}, s_{2}, s_{3}\right)$ from the unphysical sheet at $q^{2}<0$ onto the physical sheet at $q^{2}>0$ through the normal cut between the points $s_{1}^{-}\left(s_{2}, s_{3}\right)$ and $s_{1}^{+}\left(s_{2}, s_{3}\right)$.

To be more specific, we can write

$$
\lambda\left(s_{1}, s_{2}, s_{3}\right)=\left(s_{1}-s_{1}^{L}\right)\left(s_{1}-s_{1}^{R}\right),
$$

where $s_{1}^{L}=\left(\sqrt{s_{2}}-\sqrt{s_{3}}\right)^{2}$ and $s_{1}^{R}=\left(\sqrt{s_{2}}+\sqrt{s_{3}}\right)^{2}$. At $q^{2}<0$ both singularities $s_{1}^{L}$ and $s_{1}^{R}$ are located on the unphysical sheet and do not contribute to the spectral representation of the form factor. As $q^{2}$ becomes positive, $s_{1}^{R}$ moves around $s_{1}^{-}$and appears on the physical sheet. The pinching of the points $s_{1}^{R}$ and $s_{1}^{-}$occurs at $s_{2}^{0}$ such that $s_{1}^{R}\left(s_{2}^{0}\right)=s_{1}^{-}\left(s_{2}^{0}\right)$. The corresponding value $s_{2}^{0}\left(s_{3}\right)$ reads

$$
\sqrt{s_{2}^{0}}=-\frac{s_{3}+m_{1}^{2}-m_{2}^{2}}{2 \sqrt{s_{3}}}+\sqrt{\left(\frac{s_{3}+m_{1}^{2}-m_{2}^{2}}{2 \sqrt{s_{3}}}\right)^{2}+\left(m_{3}^{2}-m_{1}^{2}\right)}, \quad s_{3}<\left(m_{2}-m_{1}\right)^{2} .
$$

The spectral representation of the form factor at $q^{2}>0$ takes the form [14]

$$
f\left(q^{2}\right)=\frac{1}{16 \pi^{2}} \int_{\left(m_{1}+m_{3}\right)^{2}}^{\infty} \varphi_{2}\left(s_{2}\right) \int_{s_{1}^{-}}^{s_{1}^{+}} \varphi_{1}\left(s_{1}\right) \frac{\tilde{f}\left(s_{1}, s_{2}, q^{2}\right)}{\lambda^{1 / 2}\left(s_{1}, s_{2}, q^{2}\right)}+\theta\left(q^{2}\right) \frac{1}{8 \pi^{2}} \int_{s_{2}^{0}}^{\infty} \varphi_{2}\left(s_{2}\right) \int_{s_{1}^{R}}^{s_{1}^{-}} d s_{1} \varphi_{1}\left(s_{1}\right) \frac{\tilde{f}\left(s_{1}, s_{2}, q^{2}\right)}{\lambda^{1 / 2}\left(s_{1}, s_{2}, q^{2}\right)} .
$$

One should also take into account that if the double spectral density $f_{i}$ is singular at the point $s_{1}^{R}$ then a properly defined spectral representation contains also another kind of subtraction terms: these terms appear as the contribution of a small circle around $s_{1}^{R}$ which is the lower integration limit in the anomalous part. These subtractions have quite different nature and different form than subtractions in the spectral representations at $q^{2}<0$. The corresponding expression can be found in 15$]$.

The normal contribution dominates the form factor at small timelike and vanishes as $q^{2}=\left(m_{2}-m_{1}\right)^{2}$ while the anomalous contribution is negligible at small $q^{2}$ and steeply rises as $q^{2} \rightarrow\left(m_{2}-m_{1}\right)^{2}$.

For pseudoscalar and vector mesons with the mass $M$ built up of the constituent quarks $m_{q}$ and $m_{\bar{q}}$, the function $\varphi$ is normalized as follows [14]

$$
\frac{1}{8 \pi^{2}} \int d s \varphi^{2}(s) \frac{\lambda^{1 / 2}\left(s, m_{q}^{2}, m_{\bar{q}}^{2}\right)}{s}\left[s-\left(m_{q}-m_{\bar{q}}\right)^{2}\right]=1 .
$$


This equation is the normalization of the elastic charge form factor at $q^{2}=0$.

The meson wave function can be written in the form

$$
\varphi(s)=\frac{\pi}{\sqrt{2}} \frac{\sqrt{s^{2}-\left(m_{1}^{2}-m_{2}^{2}\right)^{2}}}{\sqrt{s-\left(m_{1}-m_{2}\right)^{2}}} \frac{1}{s^{3 / 4}} w(k), \quad k=\frac{\lambda^{1 / 2}\left(s, m_{1}^{2}, m_{2}^{2}\right)}{2 \sqrt{s}}
$$

where $w(k)$ is the ground-state $S$-wave radial wave function.

In the next sections we analyze the form factors given by the dispersion representation (47) with the spectral densities (26 31) and (41 44) and demonstrate them to have the following properties in the case of a heavy parent meson: for the transition induced by the heavy-to-heavy quark transition they satisfy the LO and NLO relations 29] of the $1 / m_{Q}$ expansion in accordance with QCD provided the functions $\varphi_{i}$ are localized near the $q \bar{q}$ threshold with the width of order $\Lambda_{Q C D}$. For the meson decay induced by the heavy-to-light quark transition they satisfy the LO relations between the form factors of the vector, axial-vector and tensor currents 32 .

\section{HEAVY-QUARK EXPANSION IN QUARK MODEL FOR HEAVY-TO-HEAVY TRANSITIONS}

In this section we consider the form factors of the dispersion quark model in the case when both $m_{2}$ and $m_{1}$ are large. We calculate the universal form factors and demonstrate that requiring the structure of the $1 / m_{Q}$ expansion in the quark model to be consistent with the structure of such expansion in QCD allows us to determine the subtraction terms.

\section{A. Soft wave function and normalization condition}

First, we need to specify the properties of the soft wave function of a heavy meson. A basic property of such soft wave function $\varphi\left(s, m_{Q}, m_{\bar{q}}, \bar{\Lambda}\right)$ is a strong peaking near the $q \bar{q}$ threshold. For elaborating the $1 / m_{Q}$ expansion, it is convenient to formulate such peaking in terms of the variable $z$ such that $s=\left(m_{Q}+m_{3}+z\right)^{2}$ (hereafter we denote the mass of the light quark as $m_{3}$ ). The region above the $q \bar{q}$ threshold which contributes to the spectral representation corresponds to $z>0$. A localization of the soft wave function in terms of $z$ means that the wave function is nonzero as $z \leq \Lambda_{Q C D}$. In the heavy meson case $m_{Q} \gg m_{3} \simeq z \simeq \bar{\Lambda}$. Let us notice that for a heavy meson the localization in terms of $z$ is equivalent to the localization in terms of the relative momentum in the meson rest frame

$$
\vec{k}^{2}=z\left(z+2 m_{3}\right)+O\left(1 / m_{Q}\right) .
$$

The normalization condition (48) which is a consequence of the vector current conservation in the full theory provides an (infinite) chain of relations in the effective theory. Namely, expanding the soft wave function in $1 / m_{Q}$ as follows

$$
\varphi\left(s, m_{Q}, m_{3}, \bar{\Lambda}\right)=\frac{\pi}{\sqrt{m_{Q}}} \phi_{0}\left(z, m_{3}, \bar{\Lambda}\right)\left[1+\frac{m_{3}}{4 m_{Q}} \chi_{1}\left(z, m_{3}, \bar{\Lambda}\right)+O\left(1 / m_{Q}^{2}\right)\right]
$$

we come to the normalization condition in the form

$$
\int d z \phi_{0}^{2}(z) \sqrt{z}\left(z+2 m_{3}\right)^{3 / 2}\left[1+\frac{m_{3}}{2 m_{Q}} \chi_{1}(z)-\frac{m_{3}}{2 m_{Q}}+\ldots\right]=1 .
$$

This exact relation is equivalent to an infinite chain of equations in different $1 / m_{Q}$ orders. Lowest order relations take the form

$$
\begin{aligned}
& \int d z \phi_{0}^{2}(z) \sqrt{z}\left(z+2 m_{3}\right)^{3 / 2}=1 \\
& \int d z \phi_{0}^{2}(z) \sqrt{z}\left(z+2 m_{3}\right)^{3 / 2} \chi_{1}(z)=1 .
\end{aligned}
$$

\section{B. The variables $\omega$ and $\bar{\omega}$}

In the description of the transition processes the dispersion formulation of the quark model has the following feature: since the underlying process is the quark transition, the relevant kinematical variable for the description of the dynamics of the process is the quark recoil $\bar{\omega}$ which is defined as follows 


$$
q^{2}=\left(m_{2}-m_{1}\right)^{2}-2 m_{1} m_{2}(\bar{\omega}-1) .
$$

The relationship between $\omega$ and $\bar{\omega}$ is given by the condition that the spectral representation for the form factor is written at fixed value of $q^{2}$ and hence

$$
q^{2}=\left(M_{1}-M_{2}\right)^{2}-2 M_{1} M_{2}(\omega-1)=\left(m_{2}-m_{1}\right)^{2}-2 m_{1} m_{2}(\bar{\omega}-1) .
$$

In the case of heavy particle transitions these quantities are related to each other as

$$
\bar{\omega}=\omega+\bar{\Lambda}\left(\frac{1}{m_{1}}+\frac{1}{m_{2}}\right)(\omega-1)+O\left(1 / m_{Q}^{2}\right) .
$$

We shall obtain the representations of the form factors as functions of the variable $\bar{\omega}$. The variables $\omega$ and $\bar{\omega}$ are different by the terms of order $1 / m_{Q}$ at nonzero recoil. On the other hand, the quark and meson zero recoil points coincide with $1 / m_{Q}^{2}$ accuracy. This means that in the analyses of the $1 / m_{Q}$ expansion at nonzero recoil the difference between the $\omega$ and $\bar{\omega}$ might be ignored in the Isgur-Wise function, but gives nontrivial contribution to the NLO form factors. At the same time, at zero recoil the difference between $\omega$ and $\bar{\omega}$ might be neglected both in the leading and next-to-leading orders. Namely, the quark model provides the expansion of the form factor in the following form

$$
\begin{aligned}
h & =h_{0}(\bar{\omega})+\frac{1}{m_{1}} h_{1}^{(1)}(\bar{\omega})+\frac{1}{m_{2}} h_{1}^{(2)}(\bar{\omega})+\ldots= \\
& =h_{0}(\omega)+h_{0}^{\prime}(\omega) \bar{\Lambda}\left(\frac{1}{m_{1}}+\frac{1}{m_{2}}\right)(\omega-1)+\frac{1}{m_{1}} h_{1}^{(1)}(\bar{\omega})+\frac{1}{m_{2}} \bar{h}_{1}^{(2)}(\bar{\omega})+\ldots
\end{aligned}
$$

As we shall see later, among the NLO form factors only $\rho_{1,2}$ are affected by the the difference between $\omega$ and $\bar{\omega}$ whereas $\xi, \xi_{3}, \chi_{2}$ are not.

\section{Relative magnitudes of the normal and the anomalous contributions}

We are going now to demonstrate that the anomalous contribution comes into the game only in close vicinity of the zero recoil point whereas beyond this region is negligible.

Let us study the behavior of the anomalous contribution in the region

$$
\bar{\omega}-1 \simeq m_{Q}^{-(2+\varepsilon)} .
$$

Introducing the variables $z_{1}$ and $z_{2}$ such that $s_{1}=\left(m_{2}+m_{3}+z_{1}\right)^{2}$ and $s_{2}=\left(m_{1}+m_{3}+z_{2}\right)^{2}$ we find that the magnitude of the anomalous contribution is controlled by the value of $z_{2}^{0}(\bar{\omega})$ such that $s_{2}^{0}=\left(m_{1}+m_{3}+z_{2}^{0}(\bar{\omega})\right)^{2}$ which is the lower boundary of the $z_{2}$ integration. If $z_{2}^{0}(\bar{\omega})$ becomes large, i.e. of the order $m_{Q}$, the anomalouis contribution is suppressed by the fall-down of the soft wave function. This suppression is at least stronger than $1 / m_{Q}^{2}$. This means that the anomalous contribution is nonvanishing only if

$$
z_{2}^{0}(\bar{\omega})=\frac{m_{1} m_{2} \sqrt{\bar{\omega}^{2}-1}+m_{1} m_{2} \bar{\omega}-m_{1}^{2}}{\sqrt{m_{1}^{2}+m_{2}^{2}-2 m_{1} m_{2} \bar{\omega}}}-m_{1}+O\left(m_{3}\right) \simeq \bar{\Lambda} .
$$

In the region $\bar{\omega}-1 \simeq m_{Q}^{-(2+\varepsilon)}$, one finds $z_{2}^{0}(\bar{\omega}) \simeq m_{Q}^{-\varepsilon / 2}$. Hence the anomalous contribution comes actually into the game only in the $O\left(1 / m_{Q}^{2}\right)$ vicinity of the zero recoil point but otherwise might be neglected. On the other hand, at the quark zero recoil point $\bar{\omega}=1$, the normal contribution vanishes and the form factor is given by the anomalous contribution.

We shall calculate the form factors in the region $\bar{\omega}-1=O(1)$ where only the normal contribution should be taken into account in leading and subleading orders.

\section{The LO analysis}

To perform the $\mathrm{LO}$ analysis of the form factors let us start with the integration measure. With $1 / m_{Q}$ accuracy it can be represented in the form

$$
\frac{1}{16 \pi^{2}} \frac{d s_{1} d s_{2} \theta\left(s_{2} \geq\left(m_{1}+m_{3}\right)^{2}\right) \theta\left(s_{1}^{-} \leq s_{1} \leq s_{1}^{+}\right)}{\lambda^{1 / 2}\left(s_{1}, s_{2}, s_{3}\right)} \simeq \frac{1}{4 \pi^{2}} d z_{2} \sqrt{z_{2}\left(z_{2}+2 m_{3}\right)} \frac{d \eta}{2} \theta\left(z_{2}\right) \theta\left(\eta^{2}<1\right),
$$


and the expression for $z_{1}$ reads

$$
z_{1}=z_{2} \bar{\omega}+m_{3}(\bar{\omega}-1)+\eta \sqrt{z_{2}\left(z_{2}+2 m_{3}\right)} \sqrt{\bar{\omega}^{2}-1}+O\left(1 / m_{Q}\right) .
$$

Let us point out that the LO integration measure is symmetric in $z_{1}$ and $z_{2}$.

Next, we need expanding the spectral densities (26 35). To this end we must take into account that under the integral $\operatorname{sign} z_{1}$ and $z_{2}$ are localized in the region $z \leq \bar{\Lambda}$ due to the soft wave functions $\phi(z)$.

In LO the kinematical coefficients 36 40 in the region $\bar{\omega}-1=O(1)$ simplify to

$$
\begin{aligned}
\lambda\left(s_{1}, s_{2}, q^{2}\right) & =4 m_{1}^{2} m_{2}^{2}\left(\bar{\omega}^{2}-1\right), \\
\alpha_{1} & =\frac{1}{m_{2}(\bar{\omega}+1)}\left[m_{3}+z_{2}+\frac{z_{2}-z_{1}}{\bar{\omega}-1}\right], \\
\alpha_{2} & =\frac{1}{m_{1}(\bar{\omega}+1)}\left[m_{3}+z_{1}+\frac{z_{1}-z_{2}}{\bar{\omega}-1}\right], \\
\beta & =\frac{1}{2}\left[m_{3}^{2}-\frac{2}{\omega+1}\left(m_{3}+z_{1}\right)\left(m_{3}+z_{2}\right)+\frac{\left(z_{1}-z_{2}\right)^{2}}{\bar{\omega}^{2}-1}\right], \\
\alpha_{11} & =\alpha_{1}^{2}+\frac{\beta}{m_{2}^{2}\left(\bar{\omega}^{2}-1\right)}, \quad \alpha_{12}=\alpha_{1} \alpha_{2}-\frac{\beta \bar{\omega}}{m_{1} m_{2}\left(\bar{\omega}^{2}-1\right)}, \\
C_{0} & =-4 m_{2}(\bar{\omega}-1), \quad B=\frac{1}{2 m_{1}}, \quad C_{1}=2 m_{1} z_{2}, \quad C_{2}=2 m_{2}\left(z_{1}+2 m_{3}\right) .
\end{aligned}
$$

One finds the LO behavior of the form factor densities (26 32) is determined by the term proportional to $\tilde{s}$. The latter reads in the LO

$$
\tilde{s} \simeq 2\left(m_{3}+\frac{z_{1}+z_{2}+2 m_{3}}{\bar{\omega}+1}\right) .
$$

The LO expression for $\tilde{f}$ takes the form

$$
\tilde{f}_{D}=(\bar{\omega}+1)\left(m_{3}+\frac{z_{1}+z_{2}+2 m_{3}}{\bar{\omega}+1}\right) .
$$

The spectral densities $\tilde{a}_{1}$ and $\tilde{g}_{0 D}$ vanish in the leading order.

Hence the LO relations (11 20) are fulfilled with the Isgur-Wise (IW) function

$$
\xi(\omega)=\int d z_{2} \phi_{0}\left(z_{2}\right) \sqrt{z_{2}\left(z_{2}+2 m_{3}\right)} \int_{-1}^{1} \frac{d \eta}{2} \phi_{0}\left(z_{1}\right)\left(m_{3}+\frac{2 m_{3}+z_{1}+z_{2}}{1+\omega}\right) .
$$

In (72) we used the equality of $\bar{\omega}$ and $\omega$ with $1 / m_{Q}$ accuracy. The normalization condition of the LO wave functions $(53)$ yields $\xi(1)=1$. For the slope of the IW function at zero recoil, $\rho^{2}=-\xi^{\prime}(1)$, one finds

$$
\rho^{2}=\frac{1}{3} \int d z \sqrt{z}\left(z+2 m_{3}\right)^{3 / 2}\left(\phi_{0}^{\prime}(z)\right)^{2} z\left(z+2 m_{3}\right)
$$

Let us point out that the subtraction terms in the spectral densities do not contribute in the LO relations. As we shall see later, they are important in the NLO analysis.

\section{E. The NLO analysis of the form factors $s, f_{1}, f_{2}, g_{1}, g_{2}$, and $g$}

First, let us concentrate on the NLO relations (11-16). It is convenient to analyze the linear combinations of the form factors which do not contain the LO contribution. These combinations are

$$
\begin{aligned}
g-s & =\frac{1}{2 \sqrt{m_{1} m_{2}}} \frac{1}{m_{1}}\left[\rho_{2}-\rho_{1}+\xi_{3}\right], \\
g_{1}+f_{1} & =\frac{1}{\sqrt{m_{1} m_{2}}}\left[-\rho_{2}+\rho_{1}+\xi_{3}\right], \\
g_{2}+f_{2} & =-\frac{m_{2}}{m_{1}} \frac{1}{\sqrt{m_{1} m_{2}}}\left[\rho_{2}-\rho_{1}+\xi_{3}\right], \\
f_{1}-2 m_{1} s & =\frac{2 \xi_{3}}{\sqrt{m_{1} m_{2}}} .
\end{aligned}
$$


The spectral densities of the form factor combinations in the l.h.s. of eqs. (74 76 read

$$
\begin{aligned}
\tilde{g}-\tilde{s} & =-\frac{2}{m_{1}} \beta, \\
\tilde{g}_{1}+\tilde{f}_{1} & =-4 \beta, \\
\tilde{g}_{2}+\tilde{f}_{2} & =4 \frac{m_{2}}{m_{1}} \beta,
\end{aligned}
$$

Comparison with the eqs. 74 76) yield the relation

$$
\rho_{1}(\omega)=\rho_{2}(\omega),
$$

For the form factor $\xi_{3}$ we come to the representation

$$
\xi_{3}(\omega)=-\int d z_{2} \phi_{0}\left(z_{2}\right) \sqrt{z_{2}\left(z_{2}+2 m_{3}\right)} \int_{-1}^{1} \frac{d \eta}{2} \phi_{0}\left(z_{1}\right) \frac{1}{2}\left[m_{3}^{2}-\frac{2}{\omega+1}\left(m_{3}+z_{1}\right)\left(m_{3}+z_{2}\right)+\frac{\left(z_{1}-z_{2}\right)^{2}}{\omega^{2}-1}\right],
$$

with $z_{1}$ given by the expression (63). In (82) we have neglected the $O\left(1 / m_{Q}\right)$ difference between $\omega$ and $\bar{\omega}$.

On the other hand, the equation (77) yields the representation for the form factor $\xi_{3}$ in a different form

$$
\begin{aligned}
\xi_{3}(\omega) & =\int d z_{2} \phi_{0}\left(z_{2}\right) \sqrt{z_{2}\left(z_{2}+2 m_{3}\right)} \int_{-1}^{1} \frac{d \eta}{2} \phi_{0}\left(z_{1}\right) \\
& \times\left[\frac{z_{2}+2 m_{3}}{\omega+1}\left(z_{2}+m_{3}+\frac{\left(z_{1}-z_{2}\right) \omega}{\omega-1}\right)-\frac{m_{3}}{2}\left(m_{3}+\frac{2 m_{3}+z_{1}+z_{2}}{1+\omega}\right)\right] .
\end{aligned}
$$

One can check that for the soft wave functions providing convergency of the integrals and nonsingular at $z=0$ the representations (82) and (83) are equivalent. At zero recoil one finds

$$
\xi_{3}(1)=\int d z_{2} \sqrt{z_{2}}\left(z_{2}+2 m_{3}\right)^{3 / 2} \phi_{0}^{2}\left(z_{2}\right) \frac{z_{2}}{3} \equiv \frac{\langle z\rangle}{3},
$$

where $\langle z\rangle$ is an average kinetic energy of the light constituent quark inside an (infinitely) heavy meson in its rest frame. It is worth noting that the function $\xi_{3}$ is positive for all $\omega$.

The universal form factor $\rho_{1}=\rho_{2}$ can be found from the expansion of $s(\omega)$ with a NLO accuracy. In this case the NLO terms in the $1 / m_{Q}$ expansions of the integration measure, the wave function, and the spectral density $\tilde{s}$ contribute. Namely, we can write for the form factor $h_{s}$ the expression

$$
\begin{aligned}
h_{s}(\omega) & =\int\left[d \mu_{0}+\frac{d \mu_{1}^{(1)}}{m_{1}}+\frac{d \mu_{1}^{(2)}}{m_{2}}\right]\left[\tilde{s}(\bar{\omega})+\frac{S_{1}}{m_{1}}+\frac{S_{2}}{m_{2}}\right] \phi_{0}\left(z_{1}\right)\left(1+\frac{m_{3}}{4 m_{1}} \chi_{1}\left(z_{1}\right)\right) \phi_{0}\left(z_{2}\right)\left(1+\frac{m_{3}}{4 m_{2}} \chi_{1}\left(z_{2}\right)\right) \\
& =\xi(\bar{\omega})+\left(\frac{1}{m_{1}}+\frac{1}{m_{2}}\right) \xi^{(1)}(\bar{\omega})+\ldots=\xi(\omega)+\left(\frac{1}{m_{1}}+\frac{1}{m_{2}}\right)\left[\xi^{(1)}(\omega)+\xi^{\prime}(\omega) \bar{\Lambda}(\omega-1)\right]+\ldots,
\end{aligned}
$$

and hence $\rho_{1}(\omega)=\xi^{(1)}(\omega)+\xi^{\prime}(\omega) \bar{\Lambda}(\omega-1)-\xi_{3}(\omega)-\frac{\bar{\Lambda}}{2} \xi(\omega)$. We do not present explicit expression for $\rho_{1}$. However, let us consider $\rho_{1}$ at zero recoil. The analysis of the anomalous contribution at $\bar{\omega}=1$ gives

$$
h_{s}(\bar{\omega}=1)=1+\left(\frac{1}{m_{1}}+\frac{1}{m_{2}}\right)\left(\frac{\bar{\Lambda}}{2}-\frac{\langle z\rangle}{3}\right)+\ldots
$$

Using the relations $h_{s}(\omega=1)=h_{s}(\bar{\omega}=1)+O\left(1 / m_{Q}^{2}\right)$ and $\xi_{3}(1)=\langle z\rangle / 3$ we obtain $\rho_{1}(1)=0$ just as required by the HQET.

\section{F. The NLO analysis of the form factor $f$}

First, let us demonstrate that the dispersion representation of the form factor $f$ requires subtraction. To this end consider the anomalous contribution at $\bar{\omega}=1$. For the form factor $f_{D}$ constructed from the spectral density $\tilde{f}_{D}$ through the unsubtracted double dispersion representation we find 1

\footnotetext{
${ }^{1}$ Hereafter we denote as $f_{\tilde{a}}$ the form factor constructed from the spectral density $\tilde{a}$ through unsubtracted double dispersion representation.
} 


$$
f_{D}(1)=2 \sqrt{M_{1} M_{2}}\left[1+\frac{1}{2}\left(\frac{1}{m_{1}}+\frac{1}{m_{2}}\right)\left(\langle z\rangle+m_{3}-\bar{\Lambda}\right)+\ldots\right]
$$

This value contradicts the Luke theorem which requires the $1 / m_{Q}$ corrections to vanish at zero recoil. Let us demonstrate that the form factor $f$ constructed from the spectral density with the included subtraction term

$$
\tilde{f}=\tilde{f}_{D}+\left(M_{1}^{2}-s_{1}+M_{2}^{2}-s_{2}\right) \tilde{g}=\tilde{f}_{D}+\left(2 p_{1} p_{2}-2 \tilde{p}_{1} \tilde{p}_{2}\right) \tilde{g}
$$

satisfies the NLO relation (19). Here $2 \tilde{p}_{1} \tilde{p}_{2}=s_{1}+s_{2}-s_{3}$.

We have noticed above that to LO and NLO the expansion of the form factor $f$ and the combination $2 p_{1} p_{2}$. $g-m_{1} \cdot g_{2}$ coincide (21). Hence checking the NLO relations for the form factor $f$ is equivalent to checking with the NLO accuracy the relation

$$
f_{D} \simeq f_{2 \tilde{p}_{1} \tilde{p}_{2} \cdot \tilde{g}}-m_{1} \cdot g_{2}
$$

The spectral density of the r.h.s. of eq. 899 can be written as

$$
2 \tilde{p}_{1} \tilde{p}_{2} \cdot \tilde{g}-m_{1} \cdot \tilde{g}_{2} \simeq 2 m_{1} m_{2}(\bar{\omega}+1) \tilde{s}+\left[2 m_{1}\left(z_{2}+m_{3}\right)+2 m_{2}\left(z_{1}+m_{3}\right)\right] \tilde{s}-8\left(m_{1}+m_{2}\right) \beta-4 m_{2}(\bar{\omega}-1) \beta .
$$

For checking the expression (89) in NLO we need the expansion of the spectral density $\tilde{s}$ in LO and NLO which has the structure

$$
\frac{1}{2} \tilde{s}=m_{1} \alpha_{2}+m_{2} \alpha_{1}+m_{3}\left(1-\alpha_{1}-\alpha_{2}\right) \equiv m_{3}+\frac{z_{1}+z_{2}+2 m_{3}}{\bar{\omega}+1}+\frac{S_{1}}{m_{1}}+\frac{S_{2}}{m_{2}}+\ldots,
$$

with

$$
S_{1}=\frac{1}{2} z_{2}\left(z_{2}+2 m_{3}\right)-z_{1} m_{3}-\left(z_{2}+2 m_{3}\right) \frac{z_{1}-z_{2}}{\bar{\omega}-1}-\frac{\left(z_{1}+z_{2}+2 m_{3}\right)^{2}}{\bar{\omega}+1}
$$

and $S_{2}$ is obtained from $S_{1}$ by replacing $z_{1}$ and $z_{2}$. The spectral density $\tilde{f}_{D}$ reads

$$
\tilde{f}_{D}=4 m_{1} m_{2}(\bar{\omega}+1)\left(m_{3}+\frac{z_{1}+z_{2}+2 m_{3}}{\bar{\omega}+1}\right)+2 m_{2} z_{2}\left(z_{2}+2 m_{3}\right)+2 m_{1} z_{1}\left(z_{1}+2 m_{3}\right)-4 m_{2}(\bar{\omega}-1) \beta .
$$

Notice that for checking the NLO relation (89) between the form factors we do not need explicit expression for the integration measure in the NLO: in the $\mathrm{LO}$ the spectral densities are equal and hence the NLO contributions from the integration measure into both sides of the eq (89) are equal too. Finally, the eq. (89) is satisfied if the following relation is valid

$$
\begin{aligned}
& \int d z_{2} \sqrt{z_{2}\left(z_{2}+2 m_{3}\right)} \phi_{0}\left(z_{2}\right) \int_{-1}^{1} \frac{d \eta}{2} \phi_{0}\left(z_{1}\right) \\
& \quad \times\left[-z_{2}\left(z_{2}+2 m_{3}\right)+2\left(z_{1}+m_{3}\right)\left(m_{3}+\frac{z_{1}+z_{2}+2 m_{3}}{\bar{\omega}+1}\right)-4 \beta+2(\bar{\omega}+1) S_{1}\right]=0,
\end{aligned}
$$

with $z_{1}$ given by (63). One can check this relation to be true for any function $\phi_{0}(z)$ regular at $z=0$. Hence, the form factor $f$ calculated with the subtracted double spectral density (88) satisfies the HQET relations in $\mathrm{LO}$ and NLO at all $\omega$.

Strictly speaking, the NLO analysis does not allow us to uniquely specify the subtraction term: namely, any spectral density of the form

$$
\tilde{f}=\tilde{f}_{D}+\left(M_{1}^{2}-s_{1}+M_{2}^{2}-s_{2}\right) \tilde{\rho}_{f}
$$

has a proper NLO behavior in accordance with (19) provided the spectral density $\tilde{\rho}_{f}$ behaves in the LO as

$$
\tilde{\rho}_{f} \simeq 2\left(m_{3}+\frac{z_{1}+z_{2}+2 m_{3}}{\bar{\omega}+1}\right) .
$$

As we demonstrate in the next section, the analysis of the heavy-to-light LO relations requires the identification $\tilde{\rho}_{f}=\tilde{g}$.

Let us notice that the form factor obtained within the light-cone approach 6 can be represented as dispersion representation with the spectral density [15]

$$
\tilde{f}_{L C}=\frac{M_{2}}{\sqrt{s_{2}}} \tilde{f}_{D}+M_{2}\left(\frac{s_{1}-s_{2}-s_{3}}{2 \sqrt{s_{2}}}-\frac{M_{1}^{2}-M_{2}^{2}-s_{3}}{2 M_{2}}\right) \frac{\tilde{a}_{1}+\tilde{a}_{2}}{2} .
$$

At zero recoil one finds

$$
f_{L C}(1)=2 \sqrt{M_{1} M_{2}}\left[1-\frac{1}{2}\left(\frac{1}{m_{1}}-\frac{1}{m_{2}}\right)\left(\langle z\rangle+m_{3}-\bar{\Lambda}\right)+\ldots\right]
$$

that contradicts the Luke theorem. 


\section{G. The NLO analysis of the form factors $a_{1,2}$ and $\chi_{2}$}

The unsubtracted spectral densities of the form factors $a_{1}$ and $a_{2}^{\prime} \equiv a_{2}-2 s$ in the LO read

$$
\begin{aligned}
& \tilde{a}_{1 D}=4\left[\left(z_{2}+2 m_{3}\right) \alpha_{1}-m_{2} \alpha_{11}(\bar{\omega}-1)\right], \\
& \tilde{a}_{2 D}^{\prime}=4\left[\frac{m_{2}}{m_{1}}\left(z_{2}+2 m_{3}\right) \alpha_{1}-m_{2} \alpha_{12}(\bar{\omega}-1)\right] .
\end{aligned}
$$

The quantity $a_{2}^{\prime}$ is more convenient than $a_{2}$ for calculations as the LO term of the heavy quark expansion of $a_{2}^{\prime}$ is zero.

The unsubtracted spectral representation for $a_{1}(\omega) m_{2}-a_{2}(\omega) m_{1}$ in combination with eq (23) gives

$$
\chi_{2}(\omega)=\xi_{3}(\omega)-\frac{1}{4} \int d z_{2} \phi_{0}\left(z_{2}\right) \sqrt{z_{2}\left(z_{2}+2 m_{3}\right)} \int_{-1}^{1} \frac{d \eta}{2} \phi_{0}\left(z_{1}\right)\left[\tilde{a}_{1} m_{2}-\tilde{a}_{2} m_{1}\right] .
$$

Substituting the representation (82) for $\xi_{3}$ we find

$$
\chi_{2}(\omega)=0 .
$$

Let us now consider the linear combination $a_{1}(\omega) m_{2}+a_{2}^{\prime}(\omega) m_{1}$. As a first step, show that the unsubtracted dispersion representation is not compatible with HQET. To this end calculate the unsubtracted form factors $a_{1 D}$ and $a_{2 D}^{\prime}$ at zero recoil:

$$
\begin{aligned}
& a_{1 D}(1)=\frac{1}{\sqrt{m_{1} m_{2}}} \frac{1}{m_{2}}\left[\frac{2}{3}\langle z\rangle+\frac{m_{3}}{2}\right], \\
& a_{2 D}^{\prime}(1)=\frac{1}{\sqrt{m_{1} m_{2}}} \frac{1}{m_{1}}\left[\frac{1}{3}\langle z\rangle+\frac{m_{3}}{2}\right],
\end{aligned}
$$

and hence

$$
a_{1 D}(1) m_{2}+a_{2 D}^{\prime}(1) m_{1}=\frac{1}{\sqrt{m_{1} m_{2}}}\left[\langle z\rangle+m_{3}\right]
$$

in contradiction with the HQET result eq. (24)

$$
\frac{\bar{\Lambda}}{\sqrt{m_{1} m_{2}}}
$$

This fact suggests a necessity of subtraction in the quantity $a_{1} m_{2}+a_{2}^{\prime} m_{1}$. Let us write the spectral density with subtraction in the form

$$
\tilde{a}_{1} m_{2}+\tilde{a}_{2}^{\prime} m_{1}=\tilde{a}_{1 D} m_{2}+\tilde{a}_{2 D}^{\prime} m_{1}+\frac{\kappa}{\bar{\omega}+1}\left(\frac{M_{1}^{2}-s_{1}}{2 \sqrt{s_{1}}}+\frac{M_{2}^{2}-s_{2}}{2 \sqrt{s_{2}}}\right) \frac{\tilde{\rho}_{a}}{2}
$$

with

$$
\tilde{\rho}_{a} \simeq 2\left(m_{3}+\frac{z_{1}+z_{2}+2 m_{3}}{\bar{\omega}+1}\right) .
$$

Then corresponding representation for the form factor reads

$$
\begin{gathered}
a_{1}(\omega) m_{2}+a_{2}^{\prime}(\omega) m_{1}=\frac{1}{4 \sqrt{m_{1} m_{2}}} \int d z_{2} \phi_{0}\left(z_{2}\right) \sqrt{z_{2}\left(z_{2}+2 m_{3}\right)} \int_{-1}^{1} \frac{d \eta}{2} \phi_{0}\left(z_{1}\right) \\
\times\left[\tilde{a}_{1 D} m_{2}+\tilde{a}_{2 D}^{\prime} m_{1}-\frac{2 \kappa}{\omega+1}\left(z_{1}+z_{2}+2 m_{3}\right)\left(m_{3}+\frac{z_{1}+z_{2}+2 m_{3}}{\omega+1}\right)\right]+\frac{\kappa \bar{\Lambda}}{2 \sqrt{m_{1} m_{2}}} \frac{\xi(\omega)}{\omega+1} .
\end{gathered}
$$

According to the HQET eq (24) this relation should be equal to

$$
\frac{1}{\sqrt{m_{1} m_{2}}}\left[\frac{2 \bar{\Lambda}}{\omega+1} \xi(\omega)-\xi_{3}(\omega) \frac{\omega-1}{\omega+1}\right]
$$


The term proportional $\xi(\omega)$ yields $\kappa=4$. One can check that this value also makes other parts of both expressions equal. Hence we arrive at the subtracted spectral density

$$
\tilde{a}_{1} m_{2}+\tilde{a}_{2}^{\prime} m_{1}=\tilde{a}_{1 D} m_{2}+\tilde{a}_{2 D}^{\prime} m_{1}+\frac{1}{\bar{\omega}+1}\left(\frac{M_{1}^{2}-s_{1}}{\sqrt{s_{1}}}+\frac{M_{2}^{2}-s_{2}}{\sqrt{s_{2}}}\right) \tilde{\rho}_{a} .
$$

The resulting spectral densities of the form factors $a_{1}$ and $a_{2}$ with the built-in subtraction terms take the form

$$
\begin{aligned}
& \tilde{a}_{1}=\tilde{a}_{1 D}+\frac{1}{(\bar{\omega}+1) m_{2}}\left(\frac{M_{1}^{2}-s_{1}}{\sqrt{s_{1}}}+\frac{M_{2}^{2}-s_{2}}{\sqrt{s_{2}}}\right) \frac{\tilde{\rho}_{a}}{2}, \\
& \tilde{a}_{2}=\tilde{a}_{2 D}+\frac{1}{(\bar{\omega}+1) m_{1}}\left(\frac{M_{1}^{2}-s_{1}}{\sqrt{s_{1}}}+\frac{M_{2}^{2}-s_{2}}{\sqrt{s_{2}}}\right) \frac{\tilde{\rho}_{a}}{2} .
\end{aligned}
$$

\section{H. The NLO analysis of $g_{0}$.}

The unsubtracted spectral density $\tilde{g}_{0 D}$ has the form

$$
\tilde{g}_{0 D} \simeq \frac{4}{\sqrt{m_{1} m_{2}}}\left[m_{2} \alpha_{1}\left(m_{3}+m_{2} \alpha_{1}+m_{1} \alpha_{2}\right)-\frac{\beta}{\bar{\omega}+1}\right] .
$$

At zero recoil one finds

$$
g_{0 D}(1)=\frac{1}{\left(m_{1} m_{2}\right)^{3 / 2}}\left[\frac{\langle z\rangle+m_{3}}{2}+\frac{\langle z\rangle}{6}\right]
$$

On the other hand, taking into account our earlier finding $\chi_{2}=0$, the HQET result 200 reads

$$
g_{0 D}(1)=\frac{1}{\left(m_{1} m_{2}\right)^{3 / 2}}\left[\frac{\bar{\Lambda}}{2}+\frac{\langle z\rangle}{6}\right] .
$$

Our experience obtained in considering $a_{1} m_{2}+a_{2}^{\prime} m_{1}$ hints that the subtraction procedure adds a term proportional at zero recoil to $\bar{\Lambda}-\langle z\rangle-m_{3}$, and hence we expect subtraction to work properly also in the case of $g_{0}$.

As a matter of fact, the spectral density

$$
\tilde{g}_{0}=\tilde{g}_{0 D}+\frac{1}{(\bar{\omega}+1) m_{1} m_{2}}\left(\frac{M_{1}^{2}-s_{1}}{\sqrt{s_{1}}}+\frac{M_{2}^{2}-s_{2}}{\sqrt{s_{2}}}\right) \frac{\tilde{\rho}_{g_{0}}}{2}
$$

satisfies the HQ expansion (20) provided the function $\tilde{\rho}_{g_{0}}$ behaves in the $\mathrm{LO}$ as

$$
\tilde{\rho}_{g_{0}} \simeq 2\left(m_{3}+\frac{z_{1}+z_{2}+2 m_{3}}{\bar{\omega}+1}\right) .
$$

Concluding this section let us summarize our main results: we have calculated the universal form factors and demonstrated the dispersion representations with relevant subtractions in the case of heavy-to-heavy transitions to reproduce the structure of the heavy-quark expansion in QCD in the leading and next-to-leading orders. However, we have not been able to fix uniquely these subtractions. As we shall see in the next section, the heavy-to-light transitions provide further restrictions on the form of the subtraction terms.

\section{HEAVY-TO-LIGHT MESON TRANSITIONS}

In this section we discuss meson decays induced by the heavy-to-light quark transitions in which case $M_{1}=$ $m_{2}+O(1)$ is large, while $M_{2} \simeq m_{1}$ is kept finite. As found by Isgur and Wise [32], in the region $\omega-1=O(1)$ the form factors of the tensor current can be expressed through the form factors of the vector and axial-vector currents in the leading $1 / m_{2}$ order as follows 


$$
\begin{aligned}
s\left(q^{2}\right) & =\frac{1}{2 M_{1}} f_{1}\left(q^{2}\right), \\
g_{2}\left(q^{2}\right) & =-2 M_{1} g\left(q^{2}\right), \\
g_{0}\left(q^{2}\right) & =\frac{1}{M_{1}}\left[2 g\left(q^{2}\right)+a_{2}\left(q^{2}\right)\right], \\
g_{1}\left(q^{2}\right) & =\frac{1}{M_{1}}\left[-f\left(q^{2}\right)+2 p_{1} p_{2} \cdot g\left(q^{2}\right)\right] .
\end{aligned}
$$

We address the two issues: (i) perform the leading order $1 / m_{2}$ expansion of the form factors and show the fulfillment of the relations (117 120) and (ii) discuss the scaling behavior of the form factors of the transition of different heavy mesons into a fixed final light state.

\section{A. The LO $1 / m_{Q}$ expansion of the form factors in the quark model}

In the case of heavy-light transitions one observes the appearance of the two scales: the light scale $M_{2} \simeq$ $m_{1} \simeq m_{3} \simeq \bar{\Lambda}$, and the heavy scale $M_{2} \simeq m_{1}$, and we may expand the form factors in inverse powers of the small parameter $\bar{\Lambda} / m_{2}$. The kinematical coefficients in the leading $\bar{\Lambda} / m_{2}$ order in the region $\bar{\omega}-1=O(1)$ simplify to

$$
\begin{aligned}
\lambda\left(s_{1}, s_{2}, q^{2}\right) & \simeq 4 m_{2}^{2}\left[\left(z_{1}+m_{3}+\bar{\omega} m_{1}\right)^{2}-s_{2}\right], \quad \beta=O(1), \\
\alpha_{1} & \simeq \frac{1}{m_{2}} \frac{\left(z_{1}+m_{3}+\bar{\omega} m_{1}\right)\left(s_{2}-m_{1}^{2}+m_{3}^{2}\right) / 2-s_{2}\left(z_{1}+m_{3}\right)}{\left(z_{1}+m_{3}+\bar{\omega} m_{1}\right)^{2}-s_{2}}=O\left(\frac{1}{m_{2}}\right), \\
\alpha_{2} & \simeq \frac{\left(z_{1}+m_{3}+\bar{\omega} m_{1}\right)\left(z_{1}+m_{3}\right)-\left(s_{2}-m_{1}^{2}+m_{3}^{2}\right) / 2}{\left(z_{1}+m_{3}+\bar{\omega} m_{1}\right)^{2}-s_{2}}=O(1), \\
\alpha_{12} & \simeq \alpha_{1} \alpha_{2}-\frac{\beta\left(z_{1}+m_{3}+\bar{\omega} m_{1}\right)}{2 m_{2}\left[\left(z_{1}+m_{3}+\bar{\omega} m_{1}\right)^{2}-s_{2}\right]}=O\left(\frac{1}{m_{2}}\right), \\
\alpha_{11} & \simeq \alpha_{1}^{2}+\frac{1}{m_{2}^{2}} \frac{\beta s_{2}}{\left[\left(z_{1}+m_{3}+\bar{\omega} m_{1}\right)^{2}-s_{2}\right]}=O\left(\frac{1}{m_{2}^{2}}\right) .
\end{aligned}
$$

For the double spectral densities $\tilde{f}_{i}$ these expressions yield the following LO relations

$$
\begin{aligned}
\tilde{s} & =\frac{1}{2 m_{2}} \tilde{f}_{2}, \\
\tilde{g}_{2} & =2 m_{2} \tilde{g}, \\
\tilde{g}_{0 D} & =\frac{1}{m_{2}}\left(2 \tilde{g}+\tilde{a}_{2 D}\right), \\
\tilde{g}_{1} & =\frac{1}{m_{2}}\left[-\tilde{f}_{D}+2 \tilde{p}_{1} \tilde{p}_{2} \cdot \tilde{g}\right],
\end{aligned}
$$

with $2 \tilde{p}_{1} \tilde{p}_{2}=s_{1}+s_{2}-s_{3}$. Notice that these relations hold also for $\bar{\omega}=O\left(m_{2}\right)$.

First two equations directly give the LO equality of the corresponding form factors. The relation (128) between the unsubtracted spectral densities is more interesting and requires the LO identity of the subtraction terms

$$
\tilde{\rho}_{a} \simeq \tilde{\rho}_{g_{0}} .
$$

The choice $\tilde{\rho}_{a}=\tilde{\rho}_{g_{0}}=\tilde{g}$ is acceptable although there are no firm backgrounds to justify this very choice.

The most informative is the relation (129): this relation not only suggests a necessity of subtraction in the form factor $f$ but also determines the subtraction term. When considering the heavy-to-heavy transitions we have found that the subtracted spectral density of the form (88)

$$
\tilde{f}=\tilde{f}_{D}+\left(M_{1}^{2}-s_{1}+M_{2}^{2}-s_{2}\right) \tilde{\rho}_{f}
$$

matches the HQET expansion if $\tilde{\rho}_{f} \simeq \tilde{g}$ in LO. The eq. (129) prescribes $\tilde{\rho}_{f}=\tilde{g}$. 


\section{B. The scaling of the form factors with $m_{Q}$}

Let us point out that in the case of the heavy-to-light transition there is no parametrical suppression of the anomalous contribution to the form factors compared with the normal one as it was in heavy-to-heavy transitions and both parts contribute on equal footings. We shall present all the results for the normal part but the same holds also for the anomalous part.

In the region $\bar{\omega}=O(1)$ one finds for the normal contribution

$$
f_{i}(\bar{\omega})=\frac{1}{\sqrt{m_{2}}} \int \frac{d s_{2} \varphi\left(s_{2}\right) \lambda^{1 / 2}\left(s_{2}, m_{1}^{2}, m_{3}^{2}\right)}{16 \pi m_{1}} \int_{-1}^{1} \frac{d \eta \sqrt{\bar{\omega}^{2}-1} \phi_{0}\left(z_{1}\right) \tilde{f}_{i}\left(z_{1}, z_{2}, m_{1}, m_{2}, m_{3}, \bar{\omega}\right)}{\sqrt{\left(m_{1}(\bar{\omega}+1)+z_{1}+z_{2}+2 m_{3}\right)\left(m_{1}(\bar{\omega}-1)+z_{1}-z_{2}\right)}}
$$

where

$$
\begin{aligned}
& s_{2}=\left(m_{1}+m_{3}+z_{2}\right)^{2}, \\
& z_{1}=z_{2} \bar{\omega}\left(1+\frac{2 m_{3}+z_{2}}{m_{1}}\right)+m_{3}(\bar{\omega}-1)+\eta \sqrt{\bar{\omega}^{2}-1} \frac{\lambda^{1 / 2}\left(s_{2}, m_{1}^{2}, m_{3}^{2}\right)}{2 m_{1}}+O\left(1 / m_{2}\right) .
\end{aligned}
$$

Using the relations (121) one finds that the spectral densities scale at large $m_{2}$ as

$$
\tilde{f}_{i}=m_{2}^{n_{i}} \rho_{i}\left(\bar{\omega}, m_{1}, m_{3}, z_{1}, z_{2}\right) .
$$

Namely,

$$
\begin{aligned}
& \tilde{f}_{1}=O(1), \quad \tilde{f}_{2}=O\left(m_{2}\right), \quad \tilde{g}=O(1), \quad \tilde{a}_{1}=O\left(1 / m_{2}\right), \quad \tilde{a}_{2}=O(1), \\
& \tilde{f}=O\left(m_{2}\right), \quad \tilde{g}_{1}=O(1), \quad \tilde{g}_{2}=O\left(m_{2}\right), \quad \tilde{g}_{0}=O\left(1 / m_{2}\right), \quad \tilde{s}=O(1) .
\end{aligned}
$$

Hence, the form factors have the scaling behaviour of the form

$$
f_{i}=m_{2}^{n_{i}-1 / 2} r_{i}\left(\bar{\omega} ; m_{1}, m_{3} ; \varphi_{2}, \phi_{0}\right) .
$$

The variables $\omega$ and $\bar{\omega}$ are connected with each other as follows

$$
\bar{\omega}=\frac{M_{2}}{m_{1}} \omega-\frac{\bar{\Lambda}}{m_{1}}+O\left(1 / m_{2}\right),
$$

and hence the ratio

$$
f_{i}(\omega) / M^{n_{i}+1 / 2}
$$

is universal for the transition of any heavy meson into a fixed light state. This behaviour reproduces the results of [32] up to the logarithmic corrections which arise from the anomalous scaling of the quark currents in QCD.

To summarize, matching the LO $1 / m_{2}$ relations between the form factors in the quark model to the corresponding relations of [32] allowed us to determine the subtraction term in the form factor $f$ and equated to each other subtraction terms in the form factors $a_{1,2}$ and $g_{0}$. 


\section{NUMERICAL ANALYSIS}

In this section we apply the derived results to the model-dependent estimates of the universal form factors.

1. First, let us study the dependence of the IW function on the parameters of the quark model. We adopt the exponential parametrization of the radial wave functions in the form $w\left(\vec{k}^{2}\right) \simeq \exp \left(-\vec{k}^{2} / 2 \beta^{2}\right)$. For obtaining the LO wave function $\phi_{0}$ we must take into account the relationship between the parameters $z$ and $\vec{k}^{2}$ which is found from the following equation

$$
\sqrt{s}=\sqrt{\vec{k}^{2}+m_{Q}^{2}}+\sqrt{\vec{k}^{2}+m_{3}^{2}}=m_{Q}+m_{3}+z .
$$

This yields the relation

$$
\vec{k}^{2}=z\left(z+2 m_{3}\right)+O\left(1 / m_{Q}\right) .
$$

Using the eq. (49) we come to the following form of the $\mathrm{LO}$ wave function

$$
\phi_{0}(z) \simeq \sqrt{\frac{z+m_{3}}{z+2 m_{3}}} \exp \left(-\frac{z\left(z+2 m_{3}\right)}{2 \beta_{0}^{2}}\right),
$$

where $\beta_{0}$ is the $\mathrm{LO}$ harmonic oscillator parameter

$$
\beta\left(m_{Q}\right)=\beta_{0}+O\left(1 / m_{Q}\right) .
$$

For calculations we need to specify $\beta_{0}$. The exact value of $\beta_{0}$ is not known but extrapolating the known values of $\beta_{D}, \beta_{B}$, and $\beta_{D^{*}}$ we find $\beta_{0} \simeq 0.4$ both in the WSB [1] and the ISGW2 [5] models. In the model of ref. 14. from the analysis of the axial-vector decay constants of pseudoscalar mesons the following approximate dependence has been proposed

$$
\beta\left(m_{Q}\right) \simeq 2.5 \frac{m_{Q} m_{3}}{m_{Q}+m_{3}}
$$

which gives $\beta_{0} \simeq 2.5 \mathrm{~m}_{3}$. Table 1 presents the relevant numerical parameters. The results of calculating the IW function through the eq. (72) are shown in Fig. 2. Table If demonstrates the values $\xi^{\prime}(1)$ calculated with the eq. (73) and the parameters of the quadratic fit of the form

$$
\xi(\omega)=1-\rho_{i}^{2}(\omega-1)+\delta_{i}(\omega-1)^{2},
$$

\begin{tabular}{|c|c|c|c|c|c|c|c|c|}
\hline Ref. & $m_{3}$ & $\beta_{0}$ & $-\xi^{\prime}(1)$ & $\rho^{2}$ & $\delta$ & $\xi_{3}(1)$ & $\rho_{\xi_{3}}^{2}$ & $\delta_{\xi_{3}}$ \\
\hline$\overline{\text { Set } 1 \text { 柿 }}$ & 0.35 & 0.4 & 1.47 & 1.34 & 0.78 & 0.077 & 1.49 & 0.88 \\
\hline Set 2 & 0.33 & 0.4 & 1.43 & 1.31 & 0.76 & 0.08 & 1.4 & 0.84 \\
\hline Set 3 , & 0.25 & 0.63 & 1.37 & 1.12 & 0.62 & 0.17 & 1.2 & 0.65 \\
\hline
\end{tabular}

obtained by interpolating the results of calculations in the range $1 \leq \omega \leq 1.5$. One can observe the value of $-\xi^{\prime}(1)$ to be considerably larger than the parameter $\rho^{2}$ obtained by the interpolation procedure.

TABLE I. Parameters of the quadratic fit to the IW function and the NLO form factor $\xi_{3}$ in various quark-model versions.

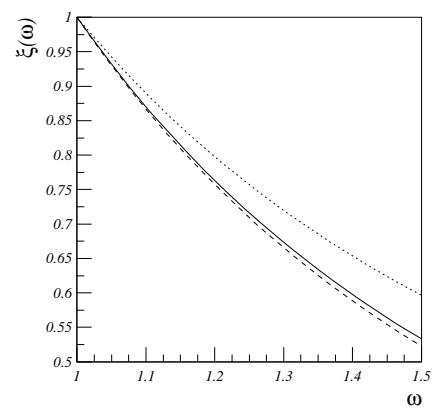

FIG. 2. The Isgur-Wise function calculated with various quark-model parameters: dashed line - set 1 , solid line - set 2 , dotted line - set 3 . 
The IW function obtained from the dispersion quark model agrees with the results of the light-cone quark model applied to the elastic $P \rightarrow P$ transition for (infinitely) heavy mesons [33] $\xi(1.5)=0.62$ and turns out to be a bit smaller than the $\mathrm{SR}$ result $\xi(1.5)=0.66$ [34].

The NLO form factor $\xi_{3}(\omega)$ is found to be very sensitive to the light-quark mass. The parameters of the quadratic fit to $\xi_{3}(\omega)$ calculated through eq. (82) in the form

$$
h_{i}(\omega)=h_{i}(1)\left[1-\rho_{i}^{2}(\omega-1)+\delta_{i}(\omega-1)^{2}\right]
$$

for various sets of the quark-model parameters are shown in Table I. One can observe an approximate relation $\xi_{3}(\omega) / \xi(\omega) \simeq 0.08 \mathrm{GeV}$ for the light quark mass $m_{3} \simeq 0.35 \mathrm{GeV}$ and $\xi_{3}(\omega) / \xi(\omega) \simeq 0.17 \mathrm{GeV}$ for the light quark mass $m_{3} \simeq 0.25 \mathrm{GeV}$ in the whole region $1 \leq \omega \leq 1.5$. This ratio is to be compared with the SR result with the $O\left(\alpha_{s}\right)$ corrections omitted [31]

$$
\xi_{3}(\omega) / \xi(\omega)=\bar{\Lambda} / 3 \simeq 0.16 \mathrm{GeV}, \quad \bar{\Lambda} \simeq 0.5 \mathrm{GeV} .
$$

2. We are in a position to estimate the higher order corrections as we can calculate the form factors at finite masses and the leading-order contribution separately. For the ISGW2 parameter set $\left(m_{c}=1.82, m_{b}=5.2\right.$,

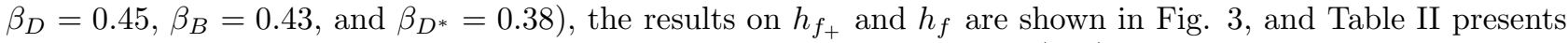
the results of interpolating in the range $1 \leq \omega \leq 1.5$ with a quadratic fit (143).

TABLE II. The form factors calculated with the parameters of ISGW2 model.

\begin{tabular}{|c||c|c|c|}
\hline \hline & $h_{f_{+}}$ & $h_{f}$ & $\xi$ \\
\hline \hline$h(1)$ & 0.93 & 0.96 & 1.0 \\
$\rho^{2}$ & 0.87 & 1.06 & 1.25 \\
$\delta$ & 0.37 & 0.53 & 0.7 \\
\hline \hline
\end{tabular}

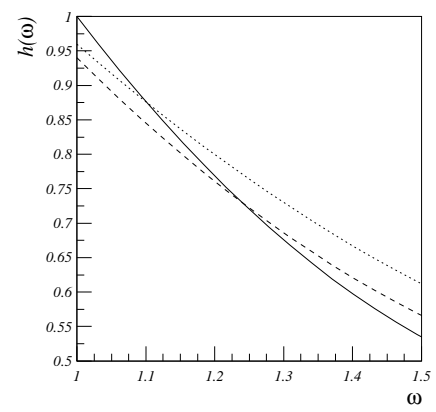

FIG. 3. The velocity-dependent form factors calculated for the ISGW2 parameters: solid line - the IW function, dashed line $-h_{f}(\omega)$, dotted line $-h_{f_{+}}(\omega)$.

The form factor $h_{f}$ can be seen to agree with the results of a combined fit to the ALEPH, ARGUS, CLEO, DELPHI, and OPAL data [35]

$$
\rho_{f}^{2}=1.07 \pm 0.20 \text {. }
$$

One observes sizeable difference between the form factors $h_{f}$ and $h_{f_{+}}$and the Isgur-Wise function both in the value at zero recoil and the slope parameter. This indicates sizeable higher order corrections. At zero recoil the $1 / m_{Q}$ corrections to $h_{f}$ vanish and writing 31

$$
h_{f}(1)=1+\delta_{1 / m^{2}}
$$

we find $\delta_{1 / m^{2}}=-0.04$ that agrees well with the value $-0.055 \pm 0.025$ [31]. At the nonzero recoil the higher-order corrections yield a more flat $\omega$-dependence of the form factor $h_{f}$ compared with the IW function such that the slope of the IW function in our model turns out to be considerably larger compared with the slope of $h_{f}$ : An approximate relation $\rho_{I W}^{2} \simeq \rho_{h_{f}}^{2}-0.2$ is found. This is different from the SR result $\rho_{I W}^{2} \simeq \rho_{h_{f}}^{2}+0.2$ [31]. Let us point out that $h^{\prime}(1)$ turns out to be considerably larger than the parameter $\rho^{2}$ of the quadratic fit obtained by the interpolation over the range $1 \leq \omega \leq 1.5$ just as in the case of the Isgur-Wise function. 


\section{CONCLUSION}

We have performed a detailed analysis of the dispersion formulation of the quark model for meson decays based on representing the transition form factors as double spectral integrals in the invariant masses of the initial and final $q \bar{q}$ pairs through the soft wave functions of the initial and final mesons. The unsubtracted double spectral densities of the form factors at spacelike momentum transfers are calculated from the Feynman graphs, and subtractions in the spectral representations are determined by considering the two following cases: (i) by performing the heavy-quark expansion of the form factors in the case of heavy-to-heavy transitions and matching them to the HQET and (ii) by considering the relations between the form factors of the vector, axial-vector and tensor currents in the case of the heavy-to-light transition and matching them to the general relations of ref. [32]. This procedure gives the spectral representations with appropriate subtractions at spacelike momentum transfers. The form factors at timelike momentum transfers are obtained by performing the analytical continuation in $q^{2}$. The analytical continuation yields the appearance of the anomalous contribution to the form factors at $q^{2}>0$. As a result, we have come to an explicit model of the form factors describing the meson decays induced by the transition between finite-mass quarks which develops the correct structure of the heavy-quark expansion. Let us emphasize that the obtained representations allow us to calculate the form factors directly at the timelike region.

Notice that for deriving the heavy-quark expansion we impose only a strong localization of the meson momentum distribution with a width of order of the confinement scale. No other constraints on the soft wave functions or numerical parameters of the model have emerged.

Our main results are:

1. We apply the method of ref. [29] to meson amplitudes of the tensor current and find the expansions of the relevant form factors in LO and NLO in HQET. The $1 / m_{Q}$ corrections in the form factor $h_{g_{+}}$are found to vanish at zero recoil just as in $h_{f_{+}}$and $h_{f}$.

2. The $1 / m_{Q}$ expansion of the soft wave function is constructed. The wave function normalization condition which is a consequence of the vector current conservation in the full theory yields an infinite chain of normalization conditions for the wave function components emerging in various $1 / m_{Q}$ orders. The LO normalization condition which is connected with the current conservation in the effective theory provides the correct normalization of the Isgur-Wise function at zero recoil.

3. In the LO all the transition form factors are represented through the Isgur-Wise function in accordance with HQET. The Isgur-Wise function is calculated through the LO component of the heavy meson wave function. The subtraction terms in spectral representations of the form factors do not contribute in LO. The anomalous contribution which emerges when the analytical continuation to the timelike region is performed comes into the game only in the $1 / m_{Q}^{2}$ vicinity of the of the zero recoil point but otherwise is suppressed by at least the second inverse power of the heavy-quark mass.

4. The NLO analysis of the $P \rightarrow P$ transitions shows that the unsubtracted dispersion representations develop $1 / m_{Q}$ structure in accordance with HQET and allows calculating $\xi_{3}$ through the LO wave function.

5. In the case of the $P \rightarrow V$ transition the unsubtracted representations for the form factors $g, g_{1}, g_{2}$, and $m_{1} a_{2}-m_{2} a_{1}$ have the NLO behavior compatible with HQET and yield the relation $\rho_{1}=\rho_{2}\left(\right.$ or $\chi_{3}=0$ ) and $\chi_{2}=0$. At the same time, the unsubtracted form factors $f, g_{0}$, and $m_{1} a_{2}+m_{2} a_{1}$ do not agree with HQET in NLO. The matching procedure allows restricting the form of the subtraction terms.

6. Analyzing the heavy-to-light transitions and requiring the fulfillment of the Isgur-Wise relations between the form factors of the tensor and vector and axial-vector currents [32] further constrains the subtraction terms. 7. We observe a discrepancy between the predictions of the various versions of the quark model on the NLO universal form factors: Namely, the analysys of the WSB model [1] performed in [30] resulted in $\rho_{1} \neq \rho_{2} \neq 0$ and $\rho_{3} \neq 0$, but $\rho_{4}=0$; the quasipotential quark model [8] predicts all the NLO form factors to be nonzero; a consistent relativistic treatment of only $q \bar{q}$ intermediate states in Feynman graphs performed in this paper gives $\rho_{1}=\rho_{2}, \rho_{3}=0$, and $\rho_{4} \neq 0$. The origin of this discrepancy between the quark models is not fully understood and should be considered in more detail.

8. The numerical results of the dispersion quark model using several parameter sets (i.e. constituent quark masses and wave functions) demonstrate a moderate dependence of the Isgur-Wise functions on the light-quark mass and the shape of the wave function. Namely, the light-quark mass $m_{3}=0.25 \div 0.35$ and the wave-function width $\beta_{0}=0.4 \div 0.65$ yield $\xi(1.5)=0.55 \div 0.6$ that is a bit smaller than the SR result $\xi(1.5)=0.66[34$.

The form factor $h_{f}$ is found to have the behavior in agreement with predictions of other models and experimental results. We observe sizeable difference between the form factor $h_{f}$ and the Isgur-Wise function both in the value at zero recoil and the slope parameter.

The size of the higher-order $1 / m_{Q}-$ corrections to $h_{f}(1), \delta_{1 / m^{2}}=-0.04$, obtained using the ISGW2 parameters agrees favorably with the sum rule estimates.

The slope of the IW function in our model turns out to be considerably larger compared with the slope of $h_{f}$ : An approximate relation $\rho_{I W}^{2} \simeq \rho_{h_{f}}^{2}-0.2$ is found. This is opposite to the SR result $\rho_{I W}^{2} \simeq \rho_{h_{f}}^{2}+0.2$ 
[31]. We would like to notice that the slope parameter is very sensitive to the interpolation procedure: namely, the value $h^{\prime}(1)$ turns out to be considerably larger than the result of the quadratic interpolation over the range $1 \leq \omega \leq 1.5$.

The dispersion quark model for the transition form factors can be further improved by performing the heavyquark expansion in higher orders and matching to the HQET order by order. It should be taken into account that the quark model effectively describes the whole $1 / m_{Q}$ series, but the short-distance corrections are not contained in the model. The inclusion of such short-distance corrections into consideration is necessary for the application of the model to the analysis of the experimental results.

\section{ACKNOWLEDGMENTS}

I am grateful to I.Narodetskii, S.Simula, B.Stech and K.Ter-Martirosyan for discussions and to M.Neubert for helpful communication. The work was supported by the RFBR under grants 95-02-04808a and 96-02-18121a.

[1] M.Wirbel, B.Stech, and M.Bauer, Z.Phys. C29, 637 (1985).

[2] T.Altomari and L.Wolfenstein, Phys.Rev. D37,681 (1988).

[3] J.G.Körner and G.A. Schuler, Z.Phys. C38, 511 (1988).

[4] N.Isgur, D.Scora, B.Grinstein, and M.Wise, Phys.Rev. D39, 799 (1989).

[5] D.Scora and N.Isgur, Phys.Rev. D52 (1995) 2783.

[6] W.Jaus, Phys.Rev. D41 (1990) 3394; ibid, D53 (1996) 1349; W.Jaus and D.Wyler, ibid, D41 (1990) 3405.

[7] B.Stech, Phys.Lett. B354 (1995) 447; hep-ph/9608297.

[8] R.N.Faustov, V.O.Galkin, Yu.A.Mishurov, Z.Phys. C66, 119 (1995); Phys.Rev. D53, 6302 (1996).

[9] G.Zoller, S.Hainzl, C.Münz, and M.Beyer, Z.Phys. C68, 103 (1995).

[10] A.Le Yaouanc, L.Oliver, O.Pene, J.C.Raynal, Phys.Lett. B365, 319 (1996).

[11] I.Grach, I.Narodetskii, and S.Simula, Phys.Lett. B385, 317 (1996).

[12] H.Y.Cheng, C.Y.Cheung, C.W.Hwang, Phys.Rev.D55, 1559 (1996).

[13] N.B.Demchuk, P.Yu.Kulikov, I.M.Narodetskii, P.J.O’Donnell, hep-ph/9701388.

[14] D.Melikhov, Phys.Rev. D53 (1996) 2460.

[15] D.Melikhov, Phys. Lett. B380 (1996) 363; ibid, B394 (1997) 385.

[16] A.Abada et al. (ELC Collaboration), Nucl.Phys. B416, 675 (1994).

[17] C.R.Allton et al. (APE Collaboration), Phys.Lett. B345, 513 (1995).

[18] J.M.Flynn et al.(UKQCD Collaboration), Nucl.Phys. B461, 327 (1996).

[19] P.Ball, V.M.Braun, and H.Dosch, Phys.Rev. D44, 3567 (1991).

[20] P.Ball, Phys.Rev. D48, 3190 (1993).

[21] S.Narison, Phys.Lett. B345, 166 (1995).

[22] P.Colangelo, F.De Fazio, P.Santorelli, and E.Scrimieri, Phys.Rev. D53, 3672 (1996).

[23] V.M.Belyaev, A.Khodjamirian, R.Rückl, Z.Phys. C60, 349 (1993).

[24] P.Ball and V.M.Braun, Phys.Rev. D55, 5561 (1997).

[25] T.M.Aliev, A. Ozpineci, M. Savci, hep-ph/9612480.

[26] C.G.Boyd, B.Grinstein, and R.F.Lebed, Nucl.Phys. B461, 493 (1996).

[27] D.Becirevic, Phys.Rev. D54, 6842 (1996).

[28] N.Isgur and M.B.Wise, Phys.Lett. B232 (1989) 113; ibid, B237 (1990) 527.

[29] M.Luke, Phys.Lett. B252 (1990) 447.

[30] M.Neubert and V.Rieckert, Nucl.Phys. B382 (1992) 97.

[31] M.Neubert, Phys.Rep. 245, 259 (1994) and references therein.

[32] N.Isgur and M.B.Wise, Phys.Rev. D42, 2388 (1990).

[33] S.Simula, Phys.Lett. B373, 193 (1996).

[34] M.Neubert, Phys.Rev. D47, 4063 (1993).

[35] M.P.Jimack, in Heavy quark physics, Proceedings of the III German-Russian Workshop, Dubna, 1996, edited by M.A.Ivanov and V.E.Lyubovitskij, p.80 (Dubna, 1996). 